\title{
On Solvability of Integro-Differential Equations
}

\section{Marta De León-Contreras ${ }^{1} \cdot$ István Gyöngy ${ }^{2}$ (D) Sizhou $\mathrm{Wu}^{3}$}

Received: 14 August 2019 / Accepted: 7 July 2020 / Published online: 25 August 2020

(C) The Author(s) 2020

\begin{abstract}
A class of (possibly) degenerate integro-differential equations of parabolic type is considered, which includes the Kolmogorov equations for jump diffusions. Existence and uniqueness of the solutions are established in Bessel potential spaces and in SobolevSlobodeckij spaces. Generalisations to stochastic integro-differential equations, arising in filtering theory of jump diffusions, will be given in a forthcoming paper.
\end{abstract}

Keywords Integro-differential equation · Bessel potential spaces · Interpolation couples

Mathematics Subject Classification (2010) Primary 45K05 · 35R09; Secondary 47G20

\section{Introduction}

We consider the equation

$$
\frac{\partial}{\partial t} u(t, x)=\mathcal{A} u(t, x)+f(t, x)
$$

on $H_{T}=[0, T] \times \mathbb{R}^{d}$ for a given $T>0$, with initial condition $u(0, x)=\psi(x)$ for $x \in \mathbb{R}^{d}$, where $\mathcal{A}$ is an integro-differential operator of the form $\mathcal{A}=\mathcal{L}+\mathcal{M}+\mathcal{N}+\mathcal{R}$, with a "zero-order" linear operator $\mathcal{R}$, a second order differential operator

$$
\mathcal{L}(t)=a^{i j}(t, x) D_{i j}+b^{i}(t, x) D_{i}+c(t, x)
$$

István Gyöngy

i.gyongy@ed.ac.uk

Marta De León-Contreras

marta.leon@uam.es

Sizhou Wu

Sizhou.Wu@ed.ac.uk

1 Departamento de Matemáticas, Facultad de Ciencias, Universidad Autónoma de Madrid, Madrid, Spain

2 School of Mathematics and Maxwell Institute, University of Edinburgh, Scotland, UK

3 School of Mathematics, University of Edinburgh, King's Buildings, Edinburgh, EH9 3JZ, UK 
and nonlocal linear operators $\mathcal{M}$ and $\mathcal{N}$ defined by

$$
\begin{gathered}
\mathcal{M}(t) \varphi(x)=\int_{Z}\left(\varphi\left(x+\eta_{t, z}(x)\right)-\varphi(x)-\eta_{t, z}(x) \nabla \varphi(x)\right) \mu(d z), \\
\mathcal{N}(t) \varphi(x)=\int_{Z}\left(\varphi\left(x+\xi_{t, z}(x)\right)-\varphi(x)\right) v(d z)
\end{gathered}
$$

for a suitable class of real-valued functions $\varphi(x)$ on $\mathbb{R}^{d}$. Here $a^{i j}, b^{i}$ and $c$ are real-valued bounded functions defined on $H_{T}, \mu$ and $v$ are $\sigma$-finite measures on a measurable space $(Z, \mathcal{Z})$. The functions $\eta$ and $\xi$ are $\mathbb{R}^{d}$-valued mapping defined on $H_{T} \times Z$. Under "zeroorder operators" we mean bounded linear operators $\mathcal{R}$ mapping the Sobolev spaces $W_{p}^{k}$ into themselves for $k=0,1,2, . ., n$ for some $n$. Examples include integral operators $\mathcal{R}(t)$ defined by

$$
\mathcal{R}(t) \varphi(x)=\int_{Z} \varphi\left(x+\zeta_{t, z}(x)\right) \lambda(d z)
$$

with appropriate functions $\zeta$ on $H_{T} \times Z$ and finite measures $\lambda$ on $\mathcal{Z}$.

Our aim is to investigate the solvability of Eq. 1.1 in Bessel potential spaces $H_{p}^{m}$ and Sobolev-Slobodeckij spaces $W_{p}^{m}$ for $p \geq 2$ and $m \in[1, \infty)$.

Such kind of equations arise, for example, as Kolmogorov equations for Markov processes given by stochastic differential equations, driven by Wiener processes and Poisson random measures, see e.g., [1, 2, 12,13] and [17]. They play important roles in studying random phenomena modelled by Markov processes with jumps, in physics, biology, engineering and finance, see e.g., [3, 8, 33, 38] and the references therein. There is a huge literature on the solvability of these equations, but in most of the publications some kind of non-degeneracy conditions on the equations, or specific assumptions on the measures $\mu$ and $v$ are assumed. Results in this direction can be found, for example, in $[12,13,17,27,29,30$, 32] and [39], and for nonlinear equations of the type (1.1), arising in the theory of stochastic control of random processes with jumps, we refer to [13] and [40]. Extensions of the $L_{p}$-theory of Krylov [18] to stochastic equations and systems of stochastic equations with integral operators of the type $\mathcal{M}$ and $\mathcal{N}$ above are developed in [6, 7, 19, 20, 28] and [31].

Note that, since with a positive constant $c_{\alpha, d}$ the fractional Laplacian operator $\Delta^{\alpha / 2}:=$ $-(-\Delta)^{\alpha / 2}$ has the integral representation

$$
\Delta^{\alpha / 2} \varphi(x)=\lim _{\varepsilon \downarrow 0} c_{\alpha, d} \int_{|z| \geq \varepsilon}(\varphi(x+z)-\varphi(x)) \frac{1}{|z|^{d+\alpha}} d z, \quad \alpha \in(0,2)
$$

for smooth functions $\varphi$ with compact support on $\mathbb{R}^{d}$, we have $\Delta^{\alpha / 2}=\mathcal{N}+\mathcal{R}-\bar{c}_{\alpha, d}$ for $\alpha \in(0,1)$ and $\Delta^{\alpha / 2}=\mathcal{M}+\mathcal{R}-\bar{c}_{\alpha, d}$ for $\alpha \in[1,2)$, where $\mathcal{M}, \mathcal{N}$ and $\mathcal{R}$ are defined in Eqs. 1.2, 1.3 and 1.4, with $\eta_{t, z}(x)=\xi_{t, z}(x)=\zeta_{t, z}(x)=z \in Z:=\mathbb{R}^{d} \backslash\{0\}$, $\mu(d z)=v(d z)=c_{\alpha, d} \mathbf{1}_{|z| \leq 1}|z|^{-d-\alpha} d z, \lambda(d z)=c_{\alpha, d} \mathbf{1}_{|z|>1}|z|^{-d-\alpha} d z$ and with

$$
\bar{c}_{\alpha, d}=c_{\alpha, d} \int_{|z|>1} \frac{1}{|z|^{d+\alpha}} d z
$$

Thus examples for Eq. 1.1 include equations with $\Delta^{\alpha / 2}, \alpha \in(0,2)$. There are many important results in the literature about fractional operators and about equations containing them, see e.g., $[4,5,42]$ and the references therein.

In this paper we are interested in the solvability of Eq. 1.1 when it can degenerate, and besides some integrability conditions, no specific conditions on the measures $\mu$ and $\nu$ are assumed. An $L_{2}$-theory of degenerate linear elliptic and parabolic PDEs is developed in [34-37]. The solvability in $L_{2}$-spaces of linear degenerate stochastic PDEs of parabolic type were first studied in [23] (see also [41]). The first existence and uniqueness theorem on 
solvability of these equations in $W_{p}^{m}$ spaces, for integers $m \geq 1$ and any $p \geq 2$, is presented in [24]. A gap in the proof of a crucial $L_{p}$-estimate in [24] is filled in, and the existence and uniqueness theorem is substantially improved in [15]. The solvability of degenerate stochastic integro-differential equations, which include the type of Eq. 1.1, are studied in [9, 25] and [26]. Existence and uniqueness theorems are obtained in Hölder spaces in [25], and in $L_{2}$-spaces in [9] and [26]. Our main result, Theorem 2.1 below, is an existence and uniqueness theorem in $L_{p}$-spaces, which generalises the main result in [9] restricted to equations without stochastic terms. To some extent Theorem 2.1 generalises also the main result, Theorem 3.3, in [26], restricted to equations without stochastic terms. Our main theorem, however, does not cover Theorem 3.3 in [26], especially that in [26] a weakly coupled system of stochastic integro-differential equations (IDEs) is considered instead of a single IDE. In this respect we note that the proof of our main result suggests that it is possible to extend it to weakly coupled systems of IDEs, but that is not considered in the present paper. Concerning solvability of Eq. 1.1 in Hölder spaces it is worth mentioning that applying our existence and uniqueness theorem in Sobolev spaces $W_{p}^{m}$ with sufficiently large $p$, and using suitable Sobolev embedding it is possible to get existence and uniqueness theorems in Hölder spaces.

A generalisation of Theorem 2.1 to stochastic integro-differential equations will be presented in a forthcoming paper.

In conclusion we introduce some notations used throughout the paper. For vectors $v=$ $\left(v^{i}\right)$ and $w=\left(w^{i}\right)$ in $\mathbb{R}^{d}$ we use the notation $v w=\sum_{i=1}^{m} v^{i} w^{i}$ and $|v|^{2}=\sum_{i}\left|v^{i}\right|^{2}$. For real-valued Lebesgue measurable functions $f$ and $g$ defined on $\mathbb{R}^{d}$ the notation $(f, g)$ means the integral of the product $f g$ over $\mathbb{R}^{d}$ with respect to the Lebesgue measure on $\mathbb{R}^{d}$. A finite list $\alpha=\alpha_{1} \alpha_{2}, \ldots, \alpha_{n}$ of numbers $\alpha_{i} \in\{1,2, \ldots, d\}$ is called a multi-number of length $|\alpha|:=n$, and the notation

$$
D_{\alpha}:=D_{\alpha_{1}} D_{\alpha_{2}} \ldots D_{\alpha_{n}}
$$

is used for integers $n \geq 1$, where

$$
D_{i}=\frac{\partial}{\partial x^{i}}, \quad \text { for } i \in\{1,2, \ldots, d\} .
$$

We use also the multi-number $\epsilon$ of length 0 , and agree that $D_{\epsilon}$ means the identity operator. For an integer $n \geq 0$ and functions $v$ on $\mathbb{R}^{d}$, whose partial derivatives up to order $n$ are functions, we use the notation $D^{n} v$ for the collection $\left\{D_{\alpha} v:|\alpha|=n\right\}$, and define

$$
\left|D^{n} v\right|^{2}=\sum_{|\alpha|=n}\left|D_{\alpha} v\right|^{2} .
$$

For differentiable functions $v=\left(v^{1}, \ldots, v^{d}\right): \mathbb{R}^{d} \rightarrow \mathbb{R}^{d}$ the notation $D v$ means the Jacobian matrix whose $j$-th entry in the $i$-th row is $D_{j} v^{i}$.

For a separable Banach space $V$ we use the notation $L_{p}([0, T], V)$ for the space of Borel functions $f:[0, T] \rightarrow V$ such that $|f|_{V}^{p}$ has finite integral with respect to the Lebesgue measure on $[0, T]$. The Borel $\sigma$-algebra on $V$ is denoted by $\mathcal{B}(V)$. The notations $C([0, T], V)$ and $C_{w}([0, T], V)$ mean the space of $V$-valued functions on $[0, T]$, which are continuous with respect to the strong topology and with respect to the weak topology, respectively, on $V$. For $m \in \mathbb{R}$ and $p \in(1, \infty)$ we use the notation $H_{p}^{m}$ for the Bessel potential space with exponent $p$ and order $m$, defined as the space of generalised functions $\varphi$ on $\mathbb{R}^{d}$ such that

$$
(1-\Delta)^{m / 2} \varphi \in L_{p} \quad \text { and } \quad|\varphi|_{H_{p}^{m}}:=\left|(1-\Delta)^{m / 2} \varphi\right|_{L_{p}}<\infty,
$$


where $\Delta=\sum_{i=1}^{d} D_{i}^{2}$, and $L_{p}$ is the space of real-valued Borel functions $f$ on $\mathbb{R}^{d}$ such that

$$
|f|_{L_{p}}^{p}:=\int_{\mathbb{R}^{d}}|f(x)|^{p} d x<\infty .
$$

For $p \in[1, \infty)$ and integers $m \geq 0$ the notation $W_{p}^{m}$ means the Sobolev space defined as the completion of $C_{0}^{\infty}=C_{0}^{\infty}\left(\mathbb{R}^{d}\right)$, the space of smooth functions with compact support on $\mathbb{R}^{d}$, in the norm

$$
|\varphi|_{W_{p}^{m}}:=\sum_{|\alpha| \leq m}\left|D_{\alpha} \varphi\right|_{L_{p}} .
$$

For integers $m \geq 0$ the space $W_{\infty}^{m}$ is the completion of $C_{b}^{\infty}$, the space of bounded functions on $\mathbb{R}^{d}$ with bounded smooth derivatives, in the norm

$$
|\varphi|_{W_{\infty}^{m}}:=\sum_{|\alpha| \leq m} \operatorname{esssup}\left|D_{\alpha} \varphi\right| .
$$

Moreover, we use $W_{q}^{-m}$ to denote the dual space of $W_{p}^{m}$, where $q$ is the conjugate exponent of $p$, i.e. $1 / p+1 / q=1$. One knows that $H_{p}^{m}$ and $W_{p}^{m}$ are the same as vector spaces, and their norms are equivalent for $p \in(1, \infty)$ and integers $m \geq 0$. When $m>0$ is not an integer, then $W_{p}^{m}$ denotes space of functions $f \in W_{p}^{\lfloor m\rfloor}$ such that

$$
\left[D_{\alpha} f\right]_{\{m\}, p}^{p}:=\int_{\mathbb{R}^{d}} \int_{\mathbb{R}^{d}} \frac{\left|D_{\alpha} f(x)-D_{\alpha} f(y)\right|^{p}}{|x-y|^{p\{m\}+d}} d x d y<\infty
$$

for every multi-index $\alpha$ of length $\lfloor m\rfloor$, where $\lfloor m\rfloor$ is the largest integer smaller than $m$, and $\{m\}=m-\lfloor m\rfloor$. When $m>0$ is not an integer, then $W_{p}^{m}$ with the norm

$$
|f|_{W_{p}^{m}}=|f|_{W_{p}^{\lfloor m\rfloor}}+\sum_{|\alpha|=\lfloor m\rfloor}\left[D_{\alpha} f\right]_{\{m\}, p}
$$

is a Banach space, called a Slobodeckij space. Derivatives are understood in the generalised sense unless otherwise noted. The summation convention with respect to repeated indices is used throughout the paper, where it is not indicated otherwise. For basic notions and results on solvability of parabolic PDEs in Sobolev spaces we refer to [21].

The paper is organised as follows. The formulation of the problem and the main result, Theorem 2.1, is in Section 2. Some technical tools and the crucial $L_{p}$ estimates are collected in Sections 3 and 4, respectively. The proof of Theorem 2.1 is given in the last section, Section 5 .

\section{Formulation of the Main Results}

Let $K$ be a fixed constant and let $\bar{\eta}$ and $\bar{\xi}$ be fixed nonnegative $\mathcal{Z}$-measurable functions on $Z$ such that

$$
K_{\bar{\eta}}^{2}:=\int_{Z} \bar{\eta}^{2}(z) \mu(d z)<\infty, \quad K_{\bar{\xi}}:=\int_{Z} \bar{\xi}(z) v(d z)<\infty .
$$

We make the following assumptions, where $p \in[2, \infty)$ and $m \geq 0$ are fixed real numbers and $\lceil m\rceil$ denotes the smallest integer which is greater than or equal to $m$.

Assumption 2.1 The derivatives of $c$ in $x \in \mathbb{R}^{d}$ up to order $\lceil m\rceil$, and the derivatives of $b^{i}$ in $x$ up to order $\max \{\lceil m\rceil, 1\}$ are Borel functions on $H_{T}$, bounded by $K$ for all $i=$ $1,2, \ldots, d$. The derivatives of $a^{i j}$ in $x$ up to order $\max \{\lceil m\rceil, 2\}$ are Borel functions on $H_{T}$ for 
$i, j=1, \ldots, d$, and are bounded by $K$. Moreover, $a^{i j}=a^{j i}$ for all $i, j=1, \ldots, d$ and for $d t \otimes d x$-almost all $(t, x) \in H_{T}$

$$
a^{i j} z^{i} z^{j} \geq 0 \text { for all }\left(z^{1}, \ldots, z^{d}\right) \in \mathbb{R}^{d} .
$$

Assumption 2.2 The function $\eta=\left(\eta^{i}\right)$ is an $\mathbb{R}^{d}$-valued $\mathcal{B}\left(H_{T}\right) \otimes \mathcal{Z}$-measurable mapping on $H_{T} \times Z$, its derivatives in $x \in \mathbb{R}^{d}$ up to order $\max \{\lceil m\rceil, 3\}$ exist and are continuous in $x$, such that

$$
|\eta| \leq \bar{\eta}, \quad\left|D^{k} \eta\right| \leq \bar{\eta} \wedge K, \quad k=1,2, \ldots, \max (\lceil m\rceil, 3)=: m_{\eta}
$$

for all $(t, x, z) \in H_{T} \times Z$, and

$$
K^{-1} \leq\left|\operatorname{det}\left(\mathbb{I}+\theta D \eta_{t, z}(x)\right)\right|
$$

for all $(t, x, z, \theta) \in H_{T} \times Z \times[0,1]$, where $\mathbb{I}$ is the $d \times d$ identity matrix and recall that $D \eta$ denotes the Jacobian matrix of $\eta$.

Remark 2.1 By Taylor's formula we have

$$
\begin{aligned}
& v(x+\eta(x))-v(x)-\eta(x) \nabla v(x)=\int_{0}^{1} \eta^{k}(x)\left(v_{k}(x+\theta \eta(x))-v_{k}(x)\right) d \theta \\
= & \int_{0}^{1} \eta^{k}(x) D_{k}(v(x+\theta \eta(x))-v(x)) d \theta-\int_{0}^{1} \theta \eta^{k}(x) \eta_{k}^{l}(x) v_{l}(x+\theta \eta(x)) d \theta
\end{aligned}
$$

for every $v \in C_{0}^{\infty}$, where to ease notation we do not write the arguments $t$ and $z$ and write $v_{k}$ instead of $D_{k} v$ for functions $v$. Due to Assumption 2.2 these equations extend to $v \in W_{p}^{1}$ for $p \geq 2$ as well. Hence after changing the order of integrals, by integration by parts we obtain

for $\varphi \in C_{0}^{\infty}$, with

$$
(\mathcal{M} v, \varphi)=-\left(\mathcal{J}^{k} v, D_{k} \varphi\right)+\left(\mathcal{J}^{0} v, \varphi\right)
$$

$$
\begin{aligned}
& \mathcal{J}^{k}(t) v(x)=\int_{0}^{1} \int_{Z} \eta^{k}\left(v\left(\tau_{\theta \eta}(x)\right)-v(x)\right) \mu(d z) d \theta, \quad k=1,2, \ldots, d, \\
& \mathcal{J}^{0}(t) v(x)=-\int_{0}^{1} \int_{Z}\left\{\sum_{k} \eta_{k}^{k}\left(v\left(\tau_{\theta \eta}(x)\right)-v(x)\right)+\theta \eta^{k}(x) \eta_{k}^{l}(x) v_{l}\left(\tau_{\theta \eta}(x)\right)\right\} \mu(d z) d \theta,
\end{aligned}
$$

where for the sake of short notation the arguments $t, z$ of $\eta$ and $\eta_{k}$ have been omitted, and

$$
\tau_{\theta \eta}(x):=x+\theta \eta_{t, z}(x) \text { for } x \in \mathbb{R}^{d}, t \in[0, T], z \in Z \text { and } \theta \in[0,1] .
$$

Assumption 2.3 The function $\xi=\left(\xi^{i}\right)$ is an $\mathbb{R}^{d}$-valued $\mathcal{B}\left(H_{T}\right) \otimes \mathcal{Z}$-measurable mapping on $H_{T} \times Z$, its derivatives in $x \in \mathbb{R}^{d}$ up to order $\max \{\lceil m\rceil, 2\}$ exist and are continuous in $x$ such that

$$
|\xi| \leq \bar{\xi}, \quad\left|D^{k} \xi\right| \leq \bar{\xi} \wedge K, \quad k=1,2, \ldots, \max (\lceil m\rceil, 2)=: m_{\xi}
$$

for all $(t, x, z) \in H_{T} \times Z$, and

$$
K^{-1} \leq\left|\operatorname{det}\left(\mathbb{I}+\theta D \xi_{t, z}(x)\right)\right|
$$

for all $(t, x, z, \theta) \in H_{T} \times Z \times[0,1]$.

Assumption 2.4 The operator $\mathcal{R}(t)$ is a linear mapping from $L_{p}\left(\mathbb{R}^{d}\right)$ into $L_{p}\left(\mathbb{R}^{d}\right)$ for every $t \in[0, T]$, such that for every $\varphi \in C_{0}^{\infty}$ the function $\mathcal{R}(t) \varphi$ is Borel measurable in $t$ and

$$
|\mathcal{R}(t) \varphi|_{W_{p}^{n}} \leq K|\varphi|_{W_{p}^{n}} \quad \text { for integers } n=0,1, \ldots,\lceil m\rceil .
$$


Remark 2.2 Clearly, there are many important examples of linear operators satisfying this condition. By Lemma 3.2 below it is not difficult to show that the operator $\mathcal{R}$ defined in Eq. 1.4 satisfies Assumption 2.4 if $\zeta=\left(\zeta^{i}\right)$ is an $\mathbb{R}^{d}$-valued $\mathcal{B}\left(H_{T}\right) \otimes \mathcal{Z}$-measurable mapping on $H_{T} \times Z$ and it is a $C^{\lceil m\rceil}$-diffeomorphism of $\mathbb{R}^{d}$ for every $(t, z) \in[0, T] \times Z$ such that

$$
\left|D^{k} \zeta\right| \leq K, \quad k=1,2, \ldots,\lceil m\rceil, \quad K^{-1} \leq\left|\operatorname{det}\left(\mathbb{I}+D \zeta_{t, z}(x)\right)\right|
$$

for all $(t, x, z) \in H_{T} \times Z$.

In the next assumption, and correspondingly in the main result below, $V_{p}^{s}$ denotes either $H_{p}^{s}$ or $W_{p}^{s}$ for every $s \geq 0$.

Assumption 2.5 We have $\psi \in V_{p}^{m}$ and $f \in L_{p}\left([0, T], V_{p}^{m}\right)$.

Using Remark 2.1 we define the notion of generalised solutions to Eq. 1.1 as follows.

Definition 2.1 An $L_{p}\left(\mathbb{R}^{d}\right)$-valued continuous function $u=u(t), t \in[0, T]$ is a generalised solution to Eq. 1.1 with initial condition $u(0)=\psi$, if $u(t) \in W_{p}^{1}\left(\mathbb{R}^{d}\right)$ for $d t$-almost every $t \in[0, T], u \in L_{p}\left([0, T], W_{p}^{1}\right)$, and

$$
(u(t), \varphi)=(\psi, \varphi)+\int_{0}^{t}\langle\mathcal{A}(s) u(s), \varphi\rangle+(f(s), \varphi) d s
$$

for every $\varphi \in C_{0}^{\infty}\left(\mathbb{R}^{d}\right)$ and $t \in[0, T]$, where

$$
\begin{gathered}
\langle\mathcal{A} u, \varphi\rangle:=-\left(a^{i j} D_{j} u, D_{i} \varphi\right)+\left(\bar{b}^{i} D_{i} u+c u, \varphi\right)-\left(\mathcal{J}^{i} u, D_{i} \varphi\right)+\left(\mathcal{J}^{0} u, \varphi\right) \\
+(\mathcal{N} u, \varphi)+(\mathcal{R} u, \varphi)
\end{gathered}
$$

with $\bar{b}^{i}=b^{i}-D_{j} a^{i j}$.

Observe that, if Assumptions 2.2 and 2.3 hold, then there is a constant $N$ such that

$$
\left|\mathcal{J}^{0}(s) v\right|_{L^{p}} \leq N|v|_{W_{p}^{1}}, \quad\left|\mathcal{J}^{k}(s) v\right|_{L^{p}} \leq N|v|_{W_{p}^{1}}, \quad|\mathcal{N}(s) v|_{L^{p}} \leq N|v|_{W_{p}^{1}},
$$

for all $v \in W_{p}^{1}$ and $s \in[0, T]$ (see Proposition 3.10 below). Thus $\langle\mathcal{A} u, \varphi\rangle$ is well-defined when Assumptions 2.1 through 2.4 are satisfied.

Theorem 2.1 Let Assumptions 2.1 through 2.5 hold with $m \geq 1$. Then Eq. 1.1 with initial condition $u(0)=\psi$ has a generalised solution $u$, which is a weakly continuous $V_{p}^{m}$-valued function, and it is strongly continuous as a $V_{p}^{s}$-valued function of $t \in[0, T]$ for any $s<m$. Moreover, there is a constant $N=N\left(K, d, m, p, T, K_{\bar{\xi}}, K_{\bar{\eta}}\right)$ such that

$$
\sup _{t \leq T}|u(t)|_{V_{p}^{s}}^{p} \leq N\left(|\psi|_{V_{p}^{s}}^{p}+\int_{0}^{T}|f(t)|_{V_{p}^{s}}^{p} d t\right) \quad \text { for } s \in[0, m] .
$$

If Assumptions 2.1 through 2.5 hold with $m=0$, then there is at most one generalised solution.

\section{3 preliminaries}

First we present some lemmas which may well be known from textbooks in analysis. Recall that we use multi-numbers $\alpha=\alpha_{1} \ldots \alpha_{n}$, where $\alpha_{j} \in\{1, \ldots, d\}$, to denote higher order 
derivatives. For a multi-number $\alpha=\alpha_{1} \ldots \alpha_{k}$ of length $k$ and a subset $\kappa$ of $\bar{k}:=\{1,2, \ldots, k\}$ we use the notation $\alpha(\kappa)$ for the multi-number $\alpha_{l_{1}} \ldots \alpha_{l_{n}}$, where $l_{1}, \ldots, l_{n}$ are the elements of $\kappa$, listed in increasing order. For short we use the notation $v_{\alpha}:=D_{\alpha} v$ for functions $v$ of $x \in \mathbb{R}^{d}$. We write $\kappa_{1} \sqcup \cdots \sqcup \kappa_{n}=\bar{k}$ for the partition of $\{1,2, . ., k\}$ into $n$ nonempty disjoint sets $\kappa_{1}, \ldots, \kappa_{n}$. Two partitions are considered different if one of the sets in one of the partitions is different from each set in the other partition. Using the above notation the chain rule for $(u(\rho))_{\alpha}:=D_{\alpha}(u(\rho))$ for functions $u: \mathbb{R}^{d} \rightarrow \mathbb{R}$ and $\rho: \mathbb{R}^{d} \rightarrow \mathbb{R}^{d}$ can be formulated as follows.

Lemma 3.1 Assume that the derivatives of $u$ and $\rho=\left(\rho^{1}, \ldots \rho^{d}\right)$ up to order $k \geq 1$ exist and are continuous functions. Then for any multi-number $\alpha=\alpha_{1} \alpha_{2} \ldots \alpha_{l}$ of length $l \in$ $\{1,2, \ldots, k\}$ we have

$$
(u(\rho))_{\alpha}=\sum_{n=1}^{l} \sum_{\kappa_{1} \sqcup \cdots \sqcup \kappa_{n}=\bar{l}} u_{i_{1} \ldots i_{n}}(\rho) \rho_{\alpha\left(\kappa_{1}\right)}^{i_{1}} \rho_{\alpha\left(\kappa_{2}\right)}^{i_{2}} \ldots \rho_{\alpha\left(\kappa_{n}\right)}^{i_{n}},
$$

where the second summation on the right-hand side means summation over the different partitions of $\bar{l}:=\{1,2, \ldots, l\}$, and for each $l$ and each partition of $\bar{l}$ there is also a summation with respect to the repeated indices $i_{j} \in\{1,2, \ldots, d\}$ for $j=1,2, \ldots, n$.

Proof One can prove this lemma by induction on $l$, and it is left for the reader as an easy exercise.

A one-to-one function, mapping $\mathbb{R}^{d}$ onto $\mathbb{R}^{d}$, is called a $C^{k}$-diffeomorphism on $\mathbb{R}^{d}$ for an integer $k \geq 1$, if the derivatives up to order $k$ of the function and its inverse are continuous. If $\rho$ is a $C^{k}$-diffeomorphism such that

$$
M \leq|\operatorname{det} D \rho| \text { and }\left|D^{i} \rho\right| \leq N \text { for } i=1,2, \ldots, k
$$

for some positive constants $M$ and $N$, then Lemma 3.1 can be extended to $u \in W_{p}^{k}$ for any $p \in[1, \infty]$.

Lemma 3.2 Let $\rho$ be a $C^{k}\left(\mathbb{R}^{d}\right)$-diffeomorphism for some $k \geq 1$ such that Eq. 3.2 holds. Then the following statements hold.

(i) There is a constant $C=C(M, N, d, p, k)$ such that for $u \in W_{p}^{l}, p \in[1, \infty]$ and $v \in W_{\infty}^{l}$

for $l=0,1,2, \ldots, k$.

$$
|u(\rho) v|_{W_{p}^{l}} \leq C|u|_{W_{p}^{l}}|v|_{W_{\infty}^{l}}
$$

(ii) For $1 \leq|\alpha| \leq k$ Eq. 3.1 holds $d x$-almost everywhere for any $u \in W_{p}^{k}, p \in[1, \infty]$.

Proof We prove (3.3) by induction on $l$, assuming that $u \in W_{p}^{l}, v \in W_{\infty}^{l}$ are smooth functions and $p \neq \infty$. For $l=0$ by the change of variable $\rho(x)=y$ and by the first inequality in Eq. 3.2 we have

$$
\begin{gathered}
|u(\rho) v|_{L_{p}}^{p} \leq \operatorname{esssup}|v|^{p} \int_{\mathbb{R}^{d}}|u(y)|^{p}\left|\operatorname{det} D \rho^{-1}(y)\right| d y \\
=\operatorname{esssup}|v|^{p} \int_{\mathbb{R}^{d}}|u(y)|^{p}\left|\operatorname{det} D \rho\left(\rho^{-1}(y)\right)\right|^{-1} d y \leq M^{-1}|u|_{L_{p}}^{p} \operatorname{esssup}|v|^{p},
\end{gathered}
$$


which proves (3.3) for $l=0$. Let $l \geq 1$ and assume that statement (i) is true for $l-1$ in place of $l$. By the Leibniz rule and the chain rule

$$
D_{i}(u(\rho) v)=u_{j}(\rho) \rho_{i}^{j} v+u(\rho) v_{i} \quad \text { for each } i=1,2, \ldots, d .
$$

Hence by the induction hypothesis and the second inequality in Eq. 3.2 we have

$$
\begin{aligned}
& \left|D_{i}(u(\rho) v)\right|_{W_{p}^{l-1}} \leq\left|u_{j}(\rho) \rho_{i}^{j} v\right|_{W_{p}^{l-1}}+\left|u(\rho) v_{i}\right|_{W_{p}^{l-1}} \\
& \leq C\left|u_{j}\right|_{W_{p}^{l-1}}\left|\rho_{i}^{j} v\right|_{W_{\infty}^{l-1}}+C|u|_{W_{p}^{l-1}}\left|v_{i}\right|_{W_{\infty}^{l-1}} \leq C(N d+1)|u|_{W_{p}^{l}}|v|_{W_{\infty}^{l}} .
\end{aligned}
$$

Thus

$$
|u(\rho) v|_{W_{p}^{l}}=\sum_{i=1}^{d}\left|D_{i}(u(\rho) v)\right|_{W_{p}^{l-1}} \leq C d(N d+1)|u|_{W_{p}^{l}}|v|_{W_{\infty}^{l}},
$$

which finishes the induction proof. When $p=\infty$ and $l=0$ then Eq. 3.3 is obvious, and by induction on $l$ we get the result as before. Clearly, the condition given by the first inequality in Eq. 3.2 is not needed in this case. Since $C_{0}^{\infty}$ is dense in $W_{p}^{l}$ when $p \neq \infty$ and $C_{b}^{\infty}$ is dense in $W_{\infty}^{l}$, we can finish the proof of (ii) by a standard approximation argument. Making use of (ii) we can get (i) also by approximating $u$ by $C_{0}^{\infty}$ functions when $p \neq \infty$ and by $C_{b}^{\infty}$ functions when $p=\infty$.

Lemma 3.3 Let $\rho$ be a $C^{k}\left(\mathbb{R}^{d}\right)$-diffeomorphism for $k \geq 1$, such that Eq. 3.2 holds. Then there are positive constants $M^{\prime}=M^{\prime}(N, d)$ and $N^{\prime}=N^{\prime}(N, M, d, k)$ such that Eq. 3.2 holds with $g:=\rho^{-1}$, the inverse of $\rho$, in place of $\rho$, with $M^{\prime}$ and $N^{\prime}$ in place of $M$ and $N$, respectively.

Proof It follows from the second estimate in Eq. 3.2 that $|\operatorname{det}(D \rho)| \leq d ! N^{d}$, and since $D g(x)=(D \rho)^{-1}(g(x))$, we have

$$
|\operatorname{det} D g(x)|=|\operatorname{det}(D \rho)(g(x))|^{-1} \geq\left(d ! N^{d}\right)^{-1},
$$

which proves the first estimate in Eq. 3.2 for $g=\rho^{-1}$ in place of $\rho$. To estimate $|D g|$ notice that $\|D g(x)\|=\lambda_{1}$, where $\|D g(x)\|$ is the operator norm of the matrix $D g(x)$, and $\lambda_{1} \geq \lambda_{2} \geq \ldots \geq \lambda_{d}>0$ are the singular values of the matrix $D g(x)$. Since $1 / \lambda_{d} \geq$ $1 / \lambda_{d-1} \geq \ldots \geq 1 / \lambda_{1}$ are the singular values of $A(x):=(D \rho)(g(x))$, we have $|\operatorname{det} A(x)|=$ $1 / \Pi_{i=1}^{d} \lambda_{i} \geq M$ and $\|A(x)\|=1 / \lambda_{d} \leq N$. Hence

$$
\left|D \rho^{-1}(x)\right| \leq K_{0}\left\|D \rho^{-1}(x)\right\|=K_{0} \lambda_{1} \leq K_{0}\left(N \lambda_{d}\right)^{d-1} \lambda_{1} \leq K_{0} N^{d-1} \prod_{i=1}^{d} \lambda_{i} \leq \frac{K_{0} N^{d-1}}{M}
$$

with a constant $K_{0}=K_{0}(d)$. To estimate $\left|D^{i} g\right|$ for $1 \leq i \leq k$ and $k>1$, we claim that for every multi-number $\alpha$ of length $i<k$ each entry $B^{r l}(\alpha)$ of the matrix $B(\alpha):=D_{\alpha} D g$ is a linear combination of products of at most $k+2$ functions, with multiplicity, taken from the set

$$
\left\{\rho_{\beta}^{j}(g), g_{\gamma}^{r}: j, r=1,2, . ., d, 1 \leq|\beta| \leq k, 1 \leq|\gamma|<k\right\}
$$

with integer coefficients, determined by $\alpha$ and $d$, where $v_{\beta}:=D_{\beta} v$ for functions $v$ and multi-numbers $\beta$. By the chain rule from $\rho(g(x))=x$ we have $A D g=I$ with $A=$ $(D \rho)(g)$. Hence, for $|\alpha|=1$

$$
D_{\alpha} D g=-A^{-1} D_{\alpha} A D g=-D g D_{\alpha} A D g=: B(\alpha) \text {. }
$$


This gives $B^{r l}(\alpha)=-g_{j}^{r} \rho_{p i}^{j}(g) g_{\alpha}^{i} g_{l}^{p}$ for $r, l=1,2, . ., d$, which proves the claim for $k=2$, and our claim follows by induction on $k$. Hence also by induction on $k$ we immediately obtain that

$$
\left|D^{i} g\right| \leq N^{\prime} \quad \text { for } 1 \leq i \leq k \text { with a constant } N^{\prime}=N^{\prime}(N, M, d, k),
$$

since we have already proved this statement for $k=1$ above.

In Section 5 we will approximate (1.1) by mollifying the data $\psi$ and $f$, the coefficients of $\mathcal{L}$ and the functions $\eta$ and $\xi$ in the variable $x \in \mathbb{R}^{d}$. It is easy to see that the mollifications of the data and the coefficients of $\mathcal{L}$ by a nonnegative $C_{0}^{\infty}$ kernel of unit integral satisfy Assumptions 2.1 and 2.5. It is less clear, however, that mollifications of $\eta$ and $\xi$ satisfy Assumptions 2.2 and 2.3. We clarify this by the help of some lemmas below. In the rest of the paper for $\varepsilon>0$ and locally integrable functions $v$ defined on $\mathbb{R}^{d}$ we use the notation $v^{(\varepsilon)}$ for the mollification of $v$, defined by

$$
v^{(\varepsilon)}(x)=S_{\varepsilon} v(x):=\varepsilon^{-d} \int_{\mathbb{R}^{d}} v(y) k((x-y) / \varepsilon) d y, \quad x \in \mathbb{R}^{d},
$$

where $k=k(x)$ is a fixed nonnegative smooth function on $\mathbb{R}^{d}$ such that $k(x)=0$ for $|x| \geq$ $1, k(-x)=k(x)$ for $x \in \mathbb{R}^{d}$, and $\int_{\mathbb{R}^{d}} k(x) d x=1$. We define also $v_{\varepsilon, \vartheta}=\vartheta v+(1-\vartheta) v^{(\bar{\varepsilon})}$ for $\varepsilon>0$ and $\vartheta \in[0,1]$.

Lemma 3.4 Let $\rho$ be a $C^{k}\left(\mathbb{R}^{d}\right)$-diffeomorphism for $k \geq 2$, such that Eq. 3.2 holds. Then there is a positive constant $\varepsilon_{0}=\varepsilon_{0}(M, N, d)$ such that $\rho_{\varepsilon, \vartheta}$ is a $C^{k}\left(\mathbb{R}^{d}\right)$-diffeomorphism for every $\varepsilon \in\left(0, \varepsilon_{0}\right)$ and $\vartheta \in[0,1]$, and Eq. 3.2 remains valid for $\rho_{\varepsilon, \vartheta}$ in place of $\rho$, with $M^{\prime \prime}=M / 2$ in place of $M$. Moreover, $\rho^{(\varepsilon)}$ is a $C^{\infty}$-diffeomorphism for $\varepsilon \in\left(0, \varepsilon_{0}\right)$.

Proof We show first that $\left|\operatorname{det} D \rho_{\varepsilon, \vartheta}\right|$ is separated away from zero for sufficiently small $\varepsilon>0$. To this end observe that if $v=\left(v^{1}, v^{2}, \ldots, v^{d}\right)$ is a Lipschitz function on $\mathbb{R}^{d}$ with Lipschitz constant $L$, and in magnitude it is bounded by a constant $K$, then for every $\varepsilon>0$ and $\vartheta \in[0,1]$

$$
\left|\Pi_{i=1}^{d} v^{i}-\Pi_{i=1}^{d} v_{\varepsilon, \vartheta}^{i}\right| \leq \sum_{i=1}^{d} K^{d-1}\left|v^{i}-v_{\varepsilon, \vartheta}^{i}\right| \leq K^{d-1} L \varepsilon .
$$

By virtue of this observation, taking into account that $D_{i} \rho^{l}$ is bounded by $N$ and it is Lipschitz continuous with a Lipschitz constant $N$, we get

$$
\left|\operatorname{det} D \rho-\operatorname{det} D \rho_{\varepsilon, \vartheta}\right| \leq d ! N^{d} \varepsilon \text {. }
$$

Thus setting $\varepsilon^{\prime}=M /\left(2 d ! N^{d}\right)$, for $\varepsilon \in\left(0, \varepsilon^{\prime}\right)$ and $\vartheta \in[0,1]$ we have

$$
\begin{aligned}
\left|\operatorname{det} D \rho_{\varepsilon, \vartheta}\right| & \geq|\operatorname{det} D \rho|-\left|\operatorname{det} D \rho-\operatorname{det} D \rho_{\varepsilon, \vartheta}\right| \\
& \geq|\operatorname{det} D \rho| / 2 \geq M / 2 .
\end{aligned}
$$

Clearly, $\rho_{\varepsilon, \vartheta}$ is a $C^{k}$ function. Hence by the implicit function theorem $\rho_{\varepsilon, \vartheta}$ is a local $C^{k}$ diffeomorphism for $\varepsilon \in\left(0, \varepsilon^{\prime}\right)$ and $\vartheta \in[0,1]$. We prove now that $\rho_{\varepsilon, \vartheta}$ is a global $C^{k}$ diffeomorphism for sufficiently small $\varepsilon$. Since by the previous lemma $\left|D \rho^{-1}\right| \leq N^{\prime}$, we have

$$
\begin{aligned}
|x-y| & \leq N^{\prime}|\rho(x)-\rho(y)| \\
& \leq N^{\prime}\left|\rho_{\varepsilon, \vartheta}(x)-\rho_{\varepsilon, \vartheta}(y)\right|+N^{\prime}\left|\rho(x)-\rho_{\varepsilon, \vartheta}(x)+\rho_{\varepsilon, \vartheta}(y)-\rho(y)\right|
\end{aligned}
$$


for all $x, y \in \mathbb{R}^{d}$ and $\varepsilon>0$ and $\vartheta \in[0,1]$. Observe that

$$
\begin{aligned}
\left|\rho(x)-\rho_{\varepsilon, \vartheta}(x)+\rho_{\varepsilon, \vartheta}(y)-\rho(y)\right| & \leq \int_{\mathbb{R}^{d}}|\rho(x)-\rho(x-\varepsilon u)+\rho(y-\varepsilon u)-\rho(y)| k(u) d u \\
& \leq \int_{\mathbb{R}^{d}} \int_{0}^{1} \varepsilon|u||\nabla \rho(x-\theta \varepsilon u)-\nabla \rho(y-\theta \varepsilon u)| k(u) d \theta d u \\
& \leq \varepsilon N|x-y| \int_{|u| \leq 1}|u| k(u) d u \leq \varepsilon N|x-y| .
\end{aligned}
$$

Thus $|x-y| \leq N^{\prime}\left|\rho_{\varepsilon, \vartheta}(x)-\rho_{\varepsilon, \vartheta}(y)\right|+\varepsilon N^{\prime} N|x-y|$. Therefore setting $\varepsilon^{\prime \prime}=1 /\left(2 N N^{\prime}\right)$, for all $\varepsilon \in\left(0, \varepsilon^{\prime \prime}\right)$ and $\vartheta \in[0,1]$ we have

$$
|x-y| \leq 2 N^{\prime}\left|\rho_{\varepsilon, \vartheta}(x)-\rho_{\varepsilon, \vartheta}(y)\right| \text { for all } x, y \in \mathbb{R}^{d},
$$

which implies $\lim _{|x| \rightarrow \infty}\left|\rho_{\varepsilon, \vartheta}(x)\right|=\infty$, i.e., the pre-image by $\rho_{\varepsilon, \vartheta}$ of any compact set is a compact set for each $\varepsilon \in\left(0, \varepsilon^{\prime \prime}\right)$ and $\vartheta \in[0,1]$. A continuous function with this property is called a proper function, and by Theorem 1 in [11] a local $C^{1}$ - diffeomorphism from $\mathbb{R}^{d}$ into $\mathbb{R}^{d}$ is a global diffeomorphism if and only if it is a proper function. Thus we have proved that $\rho_{\varepsilon, \vartheta}$ is a global $C^{k}$-diffeomorphism for $\varepsilon \in\left(0, \varepsilon_{0}\right)$ and $\vartheta \in[0,1]$, where $\varepsilon_{0}=\min \left(\varepsilon^{\prime}, \varepsilon^{\prime \prime}\right)$. Clearly, $\rho_{\varepsilon, 0}=\rho^{(\varepsilon)}$ is a $C^{\infty}$ function and hence it is a $C^{\infty}$-diffeomorphism for every $\varepsilon \in\left(0, \varepsilon_{0}\right)$.

Now we can complete the proof of the lemma by noting that since $D_{j} \rho^{(\varepsilon)}=\left(D_{j} \rho\right)^{(\varepsilon)}$, the condition $\left|D^{i} \rho\right| \leq N$ implies $\left|D^{i} \rho_{\varepsilon, \vartheta}\right| \leq N$ for any $\varepsilon>0$ and $\vartheta \in[0,1]$.

Recall the definition $\tau_{\theta \eta}$ by Eq. 2.4. Similarly, for each $t \in[0, T], \theta \in[0,1]$ and $z \in Z$ we use the notation $\tau_{\theta \xi}$ for the $\mathbb{R}^{d}$ valued function on $\mathbb{R}^{d}$, defined by

$$
\tau_{\theta \xi_{t, z}}(x)=x+\theta \xi_{t, z}(x),
$$

for $x \in \mathbb{R}^{d}$. To ease notation we will often omit the variables $t$ and $z$ of $\eta$ and $\xi$.

We can apply the above lemmas to $\tau_{\theta \eta}$ and $\tau_{\theta \xi}$ by virtue of the following proposition.

Proposition 3.5 Let Assumptions 2.2 and 2.3 hold. Then for each $t \in[0, T], \theta \in[0,1]$ and $z \in Z$ the functions $\tau_{\theta \eta}$ and $\tau_{\theta \xi}$ are $C^{k}\left(\mathbb{R}^{d}\right)$-diffeomorphisms with $m_{\eta}$ and $m_{\xi}$ in place of $k$, respectively.

Proof By the inverse function theorem $\tau_{\theta \eta}$ and $\tau_{\theta \xi}$ are local $C^{1}\left(\mathbb{R}^{d}\right)$-diffeomorphisms for each $t, \theta$ and $z$. Since

$$
\left|\eta_{t, z}(x)\right| \leq \bar{\eta}(z)<\infty, \quad\left|\xi_{t, z}(x)\right| \leq \bar{\xi}(z)<\infty,
$$

we have

$$
\lim _{|x| \rightarrow \infty}\left|\tau_{\theta \eta}(x)\right|=\lim _{|x| \rightarrow \infty}\left|\tau_{\theta \xi}(x)\right|=\infty .
$$

Hence $\tau_{\theta \eta}$ and $\tau_{\theta \xi}$ are global $C^{1}$-diffeomorphisms by Theorem 1 in [11] for each $t \in[0, T]$, $z \in Z$ and $\theta \in[0,1]$. Note that by the formula on the derivative of inverse functions a $C^{1}\left(\mathbb{R}^{d}\right)$-diffeomorphism and its inverse have continuous derivatives up to the same order. This observation finishes the proof of the proposition.

Corollary 3.6 Let Assumptions 2.2 and 2.3 hold. Then Lemmas 3.2 through 3.4 hold for $\tau_{\theta \eta}$ and $\tau_{\theta \xi}$ in place of $\rho$ and with $m_{\eta}$ and $m_{\xi}$ in place of $k$, respectively. In particular, there 
are positive constants $M=M(K, d, m), N=(K, d, m)$ and $\varepsilon_{0}=\varepsilon_{0}(K, d)$ such that for $\tilde{\rho}:=\vartheta \tau_{\theta \eta}+(1-\vartheta) \tau_{\theta \eta^{(\varepsilon)}}$ and $\bar{\rho}:=\vartheta \tau_{\theta \xi}+(1-\vartheta) \tau_{\theta \xi(\varepsilon)}$ we have

$$
\begin{gathered}
M \leq \min \left(|\operatorname{det} D \tilde{\rho}|,\left|\operatorname{det}(D \tilde{\rho})^{-1}\right|,|\operatorname{det} D \bar{\rho}|,\left|\operatorname{det}(D \bar{\rho})^{-1}\right|\right) \\
\max \left(\left|D^{k} \tilde{\rho}\right|,\left|D^{k}(\tilde{\rho})^{-1}\right|\right) \leq N, \quad \text { and } \max \left(\left|D^{l} \bar{\rho}\right|,\left|D^{l}(\bar{\rho})^{-1}\right|\right) \leq N
\end{gathered}
$$

for all $\varepsilon \in\left(0, \varepsilon_{0}\right), \theta \in[0,1], \vartheta \in[0,1],(t, x, z) \in H_{T} \times Z$, and for $k=1,2, \ldots, m_{\eta}$ and $l=1,2, \ldots, m_{\xi}$.

Lemma 3.7 Let $(S, \mathcal{S}, v)$ be a measure space with a $\sigma$-finite measure $v$, and let $g=g(s, x)$ be a $\overline{\mathcal{S} \otimes \mathcal{B}\left(\mathbb{R}^{d}\right)}$-measurable real function on $S \times \mathbb{R}^{d}$, where $\overline{\mathcal{S} \otimes \mathcal{B}\left(\mathbb{R}^{d}\right)}$ is the $v \otimes d x$ completion of the product $\sigma$-algebra $\mathcal{S} \otimes \mathcal{B}\left(\mathbb{R}^{d}\right)$. Assume that

$$
\int_{|x| \leq R} \int_{S}|g(s, x)| v(d s) d x<\infty \quad \text { for every } R>0 .
$$

Then the following statements hold.

(i) If for a multi-number $\alpha$ the derivative $D_{\alpha} g$ of $g$ in $x$ is a $\overline{\mathcal{S} \otimes \mathcal{B}\left(\mathbb{R}^{d}\right)}$-measurable function such that

$$
\int_{S} \int_{\{|x| \leq R\}}\left|D_{\alpha} g(s, x)\right| d x v(d s)<\infty
$$

for every $R>0$, then $d x$-almost everywhere

$$
D_{\alpha} \int_{S} g(s, x) v(d s)=\int_{S} D_{\alpha} g(s, x) v(d s) .
$$

(ii) If $D_{\alpha} g$ is a $\overline{\mathcal{S} \otimes \mathcal{B}\left(\mathbb{R}^{d}\right)}$-measurable function for every multi-number $\alpha,|\alpha| \leq m$, such that

$$
\int_{S}|g(s)|_{W_{p}^{m}} v(d s)<\infty
$$

then

$$
\left|\int_{S} g(s, x) v(d s)\right|_{W_{p}^{m}} \leq \int_{S}|g(s)|_{W_{p}^{m}} v(d s) .
$$

Proof Set $G(x)=\int_{S} g(s, x) v(d s)$. To prove (i) notice that by the definition of generalised derivatives and by Fubini's theorem

$$
\begin{aligned}
\int_{\mathbb{R}^{d}} D_{\alpha} G(x) \varphi(x) d x & =(-1)^{|\alpha|} \int_{\mathbb{R}^{d}} \int_{S} g(s, x) v(d s) D_{\alpha} \varphi(x) d x=\int_{S} \int_{\mathbb{R}^{d}} D_{\alpha} g(s, x) \varphi(x) d x v(d s) \\
& =\int_{\mathbb{R}^{d}} \int_{S} D_{\alpha} g(s, x) v(d s) \varphi(x) d x
\end{aligned}
$$

for every $\varphi \in C_{0}^{\infty}\left(\mathbb{R}^{d}\right)$, which implies (3.8). Hence by Hölder's inequality

$$
\left|\int_{\mathbb{R}^{d}} D_{\alpha} G(x) \varphi(x) d x\right| \leq|\varphi|_{L_{q}} \int_{S}\left|D_{\alpha} g(s)\right|_{L_{p}} v(d s)
$$

for every $\varphi \in C_{0}^{\infty}\left(\mathbb{R}^{d}\right)$, which implies

$$
\left|D_{\alpha} G\right|_{L_{p}} \leq \int_{S}\left|D_{\alpha} g(s)\right|_{L_{p}} v(d s)
$$

and Eq. 3.9 follows. 
For vectors $v=\left(v^{1}, \ldots ., v^{d}\right) \in \mathbb{R}^{d}$ we define the operators $T^{v}, I^{v}$ and $J^{v}$ by

$$
\begin{gathered}
T^{v} \varphi(x)=\varphi(x+v), \quad I^{v} \varphi(x)=\varphi(x+v)-\varphi(x), \\
J^{v} \phi(x)=\phi(x+v)-\phi(x)-v^{i} D_{i} \phi(x), \quad x \in \mathbb{R}^{d}
\end{gathered}
$$

acting on functions $\varphi$ and $\phi$ defined on $\mathbb{R}^{d}$ such that the generalised derivatives $D_{i} \phi$ exist. If $v=v(t, x, z)$ is a function of $(t, x, z) \in H_{T} \times Z$, e.g., when $v=\eta$ or $v=\xi$, then $T^{v} \varphi$, $I^{v} \varphi$ and $J^{v} \phi$ denote functions on $H_{T} \times Z$, defined by

$$
\begin{gathered}
\left(T^{\xi} \varphi\right)(t, x, z)=\varphi\left(t, x+\xi_{t, z}(x)\right) \quad\left(I^{\xi} \varphi\right)(t, x, z)=\varphi\left(t, x+\xi_{t, z}(x)\right)-\varphi(t, x), \\
\left(J^{\eta} \phi\right)(t, x, z)=\phi\left(t, x+\eta_{t, z}(x)\right)-\phi(t, x)-\eta_{t, z}^{i}(x) D_{i} \phi(t, x),
\end{gathered}
$$

for $(t, x, z)$, when $\varphi$ and $\phi$ are functions on $H_{T}$. We will often use the Taylor formulas

$$
I^{v} \varphi(x)=\int_{0}^{1} \varphi_{i}(x+\theta v) v^{i} d \theta
$$

and

$$
J^{v} \phi(x)=\int_{0}^{1}(1-\theta) \phi_{i j}(x+\theta v) v^{i} v^{j} d \theta
$$

with $\varphi_{i}:=D_{i} \varphi$ and $\phi_{i j}:=D_{i} D_{j} \phi$, which hold for every $x \in \mathbb{R}^{d}$ when $\varphi$ and $\phi$ have continuous derivatives up to first and second order, respectively. These equalities hold for $d x$-almost every $x \in \mathbb{R}^{d}$ when $\varphi \in W_{p}^{1}$ and $\phi \in W_{p}^{2}$.

Lemma 3.8 Let Assumptions 2.2 and 2.3 hold. Then $T^{\xi} \varphi(x), I^{\xi} \varphi(x)$ and $J^{\eta} \varphi(x)$ are $\mathcal{B}\left(H_{T}\right) \otimes \mathcal{Z}$-measurable functions of $(t, x, z) \in H_{T} \times Z$ for each $\varphi \in C_{0}^{\infty}$. For every multi-number $\alpha$ of length $k \leq m$ we have

$$
\begin{gathered}
\left|D_{\alpha} T^{\xi} \varphi\right|_{L_{p}} \leq N|\varphi|_{W_{p}^{k}}, \quad\left|D_{\alpha} I^{\xi} \varphi\right|_{L_{p}} \leq N \bar{\xi}(z)|\varphi|_{W_{p}^{k+1}}, \\
\left|D_{\alpha} J^{\eta} \varphi\right|_{L_{p}} \leq N \bar{\eta}^{2}(z)|\varphi|_{W_{p}^{k+2}}
\end{gathered}
$$

for $t \in[0, T], z \in Z$ and $p \in[1, \infty)$, where $N$ is a constant depending only on $d, K, m, p$.

Proof Clearly, $T^{\xi} \varphi(x), I^{\xi} \varphi(x)$ and $J^{\eta} \varphi(x)$ are $\mathcal{B}\left(H_{T}\right) \otimes \mathcal{Z}$-measurable functions by Fubini's theorem, and one can easily get estimates (3.14)-(3.15) by using Lemmas 3.1 and 3.2, together with Lemma 3.7.

Corollary 3.9 Let Assumptions 2.2 and 2.3 hold. Then for every $t, z$ the operators $T^{\xi}$, $I^{\xi}$ and $J^{\eta}$ extend to bounded linear operators from $W_{p}^{k}$ to $W_{p}^{k}$, from $W_{p}^{k+1}$ to $W_{p}^{k}$ and from $W_{p}^{k+2}$ to $W_{p}^{k}$, respectively, for $k=0,1,2, \ldots, m$, such that $T^{\xi} \varphi, I^{\xi} f$ and $J^{\eta} g$ are $\mathcal{B}([0, T]) \otimes \mathcal{Z}$-measurable $W_{p}^{k}$-valued functions of $(t, z)$ and

$$
\left|T^{\xi} \varphi\right|_{W_{p}^{k}} \leq N|\varphi|_{W_{p}^{k}}, \quad\left|I^{\xi} f\right|_{W_{p}^{k}} \leq N \bar{\xi}(z)|f|_{W_{p}^{k+1}}, \quad\left|J^{\eta} g\right|_{W_{p}^{k}} \leq N \bar{\eta}^{2}(z)|g|_{W_{p}^{k+2}}
$$

for all $\varphi \in W_{p}^{k}, f \in W_{p}^{k+1}$ and $g \in W_{p}^{k+2}$.

Proposition 3.10 Under Assumptions 2.1, 2.2 and 2.3 for every integer $k \in[1, m]$ we have

$$
\begin{gathered}
|\mathcal{L}(t) v|_{W_{p}^{k-2}} \leq N|v|_{W_{p}^{k}}, \quad|\mathcal{M}(t) v|_{W_{p}^{k-2}} \leq N|v|_{W_{p}^{k}}, \quad|\mathcal{N}(t) v|_{W_{p}^{k-1}} \leq N|v|_{W_{p}^{k}} \\
\left|\mathcal{J}^{l}(t) v\right|_{W_{p}^{k-1}} \leq N|v|_{W_{p}^{k}} \quad l=0,1,2, \ldots, d
\end{gathered}
$$

for all $v \in W_{p}^{k}$ and $t \in[0, T]$, where $\mathcal{J}^{l}$ for $l=0,1, \ldots, d$ are defined by Eqs. 2.2-2.3 and $N$ is a constant, depending only on $d, m, p, K, T, K_{\bar{\eta}}$ and $K_{\bar{\xi}}$. 
Proof It is sufficient to prove the proposition for $v \in C_{0}^{\infty}$. Then clearly, the statement on $\mathcal{L}$ with a constant $N=N(d, K, T, m, p)$ is obvious. By Taylor's formula

$\mathcal{M} v(x)=\int_{Z}(v(x+\eta)-v(x)-\eta \nabla v(x)) \mu(d z)=\int_{Z} \int_{0}^{1}(1-\theta) v_{i j}(x+\theta \eta) \eta^{i} \eta^{j} d \theta \mu(d z)$.

Hence, due to Assumption 2.2, by Lemma 3.2 for $k \in[2, m]$ we get

$$
|\mathcal{M} v|_{W_{p}^{k-2}} \leq \int_{Z} \int_{0}^{1}\left|v_{i j}(\cdot+\theta \eta)\right|_{W_{p}^{k-2}} \bar{\eta}^{2} d \theta \mu(d z) \leq|v|_{W_{p}^{k}} \int_{Z} \bar{\eta}^{2}(z) \mu(d z),
$$

which proves (3.16) for $\mathcal{M}$ when $k \geq 2$. For every $\varphi \in C_{0}^{\infty}$ by using Taylor's formula as above, then changing variable $y=x+\theta \eta$ and integrating by parts, by Hölder's inequality and Lemma 3.2 we have

a positive constant $N=N\left(K, p, d, K_{\bar{\eta}}\right)$ such that

$$
(\mathcal{M} v, \varphi) \leq N|v|_{W_{p}^{1}}|\varphi|_{W_{q}^{1}}
$$

for any $v \in W_{p}^{1}$ and $\varphi \in C_{0}^{\infty}$, which proves (3.16) for $\mathcal{M}$ when $k=1$. For $\mathcal{N}$ we have

$$
\mathcal{N} v(x)=\int_{Z}\left(v\left(x+\xi_{t, z}(x)-v(x)\right) v(d z)=\int_{Z} \int_{0}^{1} \xi_{t, z}(x) \nabla v\left(x+\theta \xi_{t, z}(x)\right) d \theta v(d z) .\right.
$$

Proceeding as before, using Assumption 2.3 we get Eq. 3.16 for $\mathcal{N}$. Estimates (3.17) can be proved similarly.

Lemma 3.11 Let Assumptions 2.2 and 2.3 hold with $m=0$. Then for $t \in[0, T]$ and $z \in Z$

$$
\begin{aligned}
& \int_{\mathbb{R}^{d}} I^{\xi} \varphi(x) d x \leq N \bar{\xi}(z)|\varphi|_{L_{1}}, \\
& \int_{\mathbb{R}^{d}} J^{\eta} \phi(x) d x \leq N \bar{\eta}^{2}(z)|\phi|_{L_{1}}
\end{aligned}
$$

for $\varphi \in W_{1}^{1}$ and $\phi \in W_{1}^{2}$ with a constant $N=N(K, d)$.

Proof The proof of Eq. 3.19 is given in [9]. For the convenience of the reader we prove both estimates here. We may assume that $\varphi, \phi \in C_{0}^{\infty}$. For each $(t, z, \theta) \in[0, T] \times Z \times[0,1]$ let $\pi_{t, z, \theta}^{-1}$ and $\tau_{t, z, \theta}^{-1}$ denote the inverse of the functions $x \rightarrow x+\theta \xi_{t, z}(x)$ and $x \rightarrow x+\theta \eta_{t, z}(x)$, respectively. Using Eqs. 3.12 and 3.13 by change of variables we have

$$
\begin{aligned}
\int_{\mathbb{R}^{d}} I^{\xi} \varphi(x) d x & =\int_{0}^{1} \int_{\mathbb{R}^{d}} \nabla \varphi(x) \chi_{t, z, \theta}(x) d x d \theta \\
\int_{\mathbb{R}^{d}} J^{\eta} \phi(x) d x & =\int_{0}^{1} \int_{\mathbb{R}^{d}}(1-\theta) D_{i j} \phi(x) \varrho_{t, z, \theta}^{i j}(x) d x d \theta
\end{aligned}
$$

with

$$
\begin{aligned}
& \chi_{t, \theta, z}(x)=\xi_{t, z}\left(\pi_{t, z, \theta}^{-1}(x)\right)\left|\operatorname{det} D \pi_{t, z, \theta}^{-1}(x)\right|, \\
& \varrho_{t, z, \theta}^{i j}(x)=\eta_{t, z}^{i}\left(\tau_{t, z, \theta}^{-1}(x)\right) \eta_{t, z}^{j}\left(\tau_{t, z, \theta}^{-1}(x)\right)\left|\operatorname{det} D \tau_{t, z, \theta}^{-1}(x)\right| .
\end{aligned}
$$

Due to Assumptions 2.2 and 2.3, using Corollary 3.6 we have a constant $N=N(K, d)$ such that

$$
\left|D \chi_{t, \theta, z}(x)\right| \leq N \bar{\xi}(z), \quad\left|D_{i j} \varrho_{t, z, \theta}^{i j}(x)\right| \leq N \bar{\eta}^{2}(z)
$$

for all $(t, z, \theta) \in[0, T] \times Z \times[0,1]$. Thus from Eqs. 3.20 and 3.21 by integration by parts we get (3.18) and (3.19). 
Next we present a special case of Theorem 2.1 from [22] on the $L_{p}$-norm of semimartingales with values in Sobolev spaces, where we use the notation $D_{\alpha}^{*}=-D_{k}$ for $\alpha=k=1,2, \ldots, d$, and $D_{0}^{*}=D_{0}$ stands for the identity operator.

Lemma 3.12 Let $\psi \in L_{p}\left(\mathbb{R}^{d}\right), u \in L_{p}\left([0, T], W_{p}^{1}\left(\mathbb{R}^{d}\right)\right)$ and $f^{\alpha} \in L_{p}\left([0, T], L_{p}\left(\mathbb{R}^{d}\right)\right)$ for some $p \geq 2$, for $\alpha=0,1, \ldots, d$, such that for each $\varphi \in C_{0}^{\infty}$

$$
\int_{\mathbb{R}^{d}} u(t) \varphi d x=\int_{\mathbb{R}^{d}} \psi \varphi d x+\int_{0}^{t} \int_{\mathbb{R}^{d}} f^{\alpha}(s) D_{\alpha}^{*} \varphi d x d s \quad d t \text {-almost everywhere. }
$$

Then, there is an $L_{p}\left(\mathbb{R}^{d}\right)$-valued continuous function $\tilde{u}$ such that $\tilde{u}(t)=u(t)$ for $d t$-a.e., and

$$
\left|\tilde{u}_{t}\right|_{L_{p}}^{p}=|\psi|_{L_{p}}^{p}+\int_{0}^{t} \int_{\mathbb{R}^{d}} p|u(s)|^{p-2} u(s) f^{0}(s)-p(p-1)|u(s)|^{p-2} D_{i} u(s) f^{i}(s) d x d s
$$

for all $t \in[0, T]$, where the repeated index $i$ means summation over $i=1,2, \ldots, d$.

The next lemma is a vector-valued version of a special case of Lemma 5.1 from [22]. Its proof is a simple exercise left for the reader.

Lemma 3.13 Let $\psi^{\alpha} \in L_{p}, u^{\alpha} \in L_{p}\left([0, T], W_{p}^{1}\left(\mathbb{R}^{d}\right)\right)$ and $f^{\alpha} \in L_{p}\left([0, T], L_{p}\left(\mathbb{R}^{d}\right)\right)$ for some $p \geq 2$, for $\alpha \in A$ for a finite index set $A$, such that for each $\varphi \in C_{0}^{\infty}$ and $\alpha \in A$

$$
\int_{\mathbb{R}^{d}} u^{\alpha}(t) \varphi d x=\int_{\mathbb{R}^{d}} \psi^{\alpha} \varphi d x+\int_{0}^{t} \int_{\mathbb{R}^{d}} f^{\alpha}(s) \varphi d x d s \quad d t \text {-almost everywhere. }
$$

Then for every $\alpha \in A$ there is an $L_{p}\left(\mathbb{R}^{d}\right)$-valued continuous function $\tilde{u}^{\alpha}$ on $[0, T]$, such that $\tilde{u}^{\alpha}(t)=u^{\alpha}(t)$ for $d t$-almost every $t \in[0, T]$, and

$$
\left|\tilde{u}_{t}\right|_{L_{p}}^{p}=|\psi|_{L_{p}}^{p}+\sum_{\alpha \in A} \int_{0}^{t} \int_{\mathbb{R}^{d}} p|u(s)|^{p-2} u^{\alpha}(s) f^{\alpha}(s) d x d s
$$

holds for all $t \in[0, T]$, where $|\tilde{u}|:=\left(\sum_{\alpha}\left(\tilde{u}^{\alpha}\right)^{2}\right)^{1 / 2}$ and $|u|:=\left(\sum_{\alpha}\left(u^{\alpha}\right)^{2}\right)^{1 / 2}$.

We will also make use of the following lemma from [22].

Lemma 3.14 Let $(S, \mathcal{S}, v)$ be a measure space, and let $\left\{v_{n}\right\}_{n \in \mathbb{N}}$ be a sequence of realvalued $\mathcal{S}$-measurable functions defined on $S$ such that such that $v_{n} \rightarrow v$ in the measure $v$, and

$$
\int_{S}\left|v_{n}\right|^{r} d v \rightarrow \int_{S}|v|^{r} d v
$$

for some $r>0$. Then $\int\left|v_{n}-v\right|^{r} d v \rightarrow 0$ as $n \rightarrow \infty$.

To prove Lemma 4.2 below, we use an estimate from [37] for nonnegative quadratic forms (see Lemma 1.7.1 therein), which, in a slightly more general setting as in [37], can be formulated as follows.

Lemma 3.15 Let be $a=\left(a^{i j}(x)\right)$ be a function, mapping $\mathbb{R}^{d}$ into the set of nonnegative definite $n \times n$-matrices with real entries for an integer $n \geq 1$. Assume that the generalised second order partial derivatives of $\left(a^{i j}\right)$ are functions, in magnitude bounded by a constant 
L. Then for dx-almost every $x \in \mathbb{R}^{d}$ we have

$$
\left|D_{l} a^{i j} V^{i j}\right|^{2} \leq N a^{i j} V^{i k} V^{j k}, \quad l=1,2, \ldots, d
$$

for all symmetric $n \times n$ matrices $V=\left(V^{i j}\right)$, with a constant $N$ depending only on $L$ and $d$.

Proof Though this lemma is known, for the convenience of the readers we present a proof of it here. Since $a(x)$ is a nonnegative symmetric matrix for every $x \in \mathbb{R}^{d}$, with second order partial derivatives bounded in magnitude by $L$, one knows from [10] (or see, e.g., Proposition IV.6.2 in [16]), the existence of a function $\sigma$, mapping $\mathbb{R}^{d}$ into the space of real-valued $n \times n$-symmetric matrices, such that $\sigma \sigma^{*}=\sigma^{2}=a$ and $|D \sigma| \leq C$ for $d x$ almost every $x \in \mathbb{R}^{d}$ with a constant $C=C(L, d)$. Thus, using the notation $f_{l}:=D_{l} f$ for functions $f$ on $\mathbb{R}^{d}$, and $\operatorname{tr}(b)$ for the trace of $n \times n$ matrices $b$, we have

$$
a_{l}:=D_{l}\left(\sigma^{2}\right)=\sigma_{l} \sigma+\sigma \sigma_{l},
$$

and by using elementary properties of the trace of matrices and of their products, we obtain

$$
\begin{gathered}
\left|a_{l}^{i j} V^{i j}\right|^{2}=\left|\operatorname{tr}\left(a_{l} V\right)\right|^{2}=\left|\operatorname{tr}\left(\sigma_{l} \sigma V\right)+\operatorname{tr}\left(\sigma \sigma_{l} V\right)\right|^{2}=4\left|\operatorname{tr}\left(\sigma_{l} \sigma V\right)\right|^{2} \\
\leq 4 \operatorname{tr}\left(\sigma_{l} \sigma_{l}^{*}\right) \operatorname{tr}\left(\sigma V(\sigma V)^{*}\right) \leq 4 C \operatorname{tr}(V a V)=4 C a^{i j} V^{i k} V^{j k}, \quad(d x-\text { a.e. }),
\end{gathered}
$$

that finishes the proof of the lemma.

\section{$4 L^{p}$ Estimates}

We are going to obtain the following crucial $L_{p}$-estimate if $p=2^{k}$ for an integer $k \geq 1$.

Theorem 4.1 Let Assumptions 2.1, 2.2 and 2.3 hold with an integer $m \geq 0$. Assume $\mathcal{R}(t)=$ 0 for all $t \in[0, T]$. Let $p=2^{k}$ for an integer $k \geq 1$. Then for $n=0,1, \ldots, m$

$$
\sum_{|\alpha|=n}\left(\left|D^{n} v\right|^{p-2} D_{\alpha} v, D_{\alpha} \mathcal{A} v\right) \leq N|v|_{W_{p}^{n}}^{p}
$$

for all $v \in W_{p}^{n+2}$ with a constant $N=N\left(d, p, m, K, K_{\bar{\xi}}, K_{\bar{\eta}}\right)$.

We prove this theorem after some lemmas.

Lemma 4.2 Let Assumption 2.1 hold. Then for $p \geq 2$ and $n=0,1, \ldots, m$

$$
Q_{n, p}(v):=\sum_{|\alpha|=n}\left(\left|D^{n} v\right|^{p-2} D_{\alpha} v, D_{\alpha} \mathcal{L} v\right) \leq N|v|_{W_{p}^{n}}^{p}
$$

for all $v \in W_{p}^{n+2}$ with a constant $N=N(d, p, m, K)$.

Proof This lemma can be obtained from general estimates given in [15]. Here we give a direct proof of it. For functions $g$ and $h$ on $\mathbb{R}^{d}$ we write $g \sim h$ if they have identical integrals over $\mathbb{R}^{d}$, and we write $g \preceq h$ if $g \sim h+\tilde{h}$ such that the integral of $\tilde{h}$ over $\mathbb{R}^{d}$ can be estimated by the right-hand side of Eq. 4.1. Consider first the case $n=0$. It is easy to see that

$$
p|v|^{p-2} v \mathcal{L} v \preceq p|v|^{p-2} v\left(a^{i j} v_{i j}+b^{i} v_{i}\right)
$$




$$
\begin{aligned}
& \sim-p(p-1)|v|^{p-2} a^{i j} v_{i} v_{j}-a_{j}^{i j}\left(|v|^{p}\right)_{i}+\left(|v|^{p}\right)_{i} b^{i} \\
& \sim-p(p-1)|v|^{p-2} a^{i j} v_{i} v_{j}+\left(a_{j i}^{i j}-b_{i}^{i}\right)|v|^{p} \\
& \preceq-p(p-1)|v|^{p-2} a^{i j} v_{i} v_{j},
\end{aligned}
$$

where, and later on, we use the notation $g_{\alpha}:=D_{\alpha} g$ for functions $g$ over $\mathbb{R}^{d}$ and multinumbers $\alpha=\alpha_{1} \ldots \alpha_{n}$. This by virtue of Assumption 2.1 proves (4.1) when $n=0$. Let us now estimate $Q$ when $n \geq 1$. Then it is easy to see that

$$
\begin{gathered}
A:=p\left|D^{n} v\right|^{p-2} \sum_{|\alpha|=n} v_{\alpha} D_{\alpha} \mathcal{L} v \\
\preceq p\left|D^{n} v\right|^{p-2} \sum_{|\alpha|=n}\left(v_{\alpha} a^{i j} v_{\alpha i j}+\sum_{l=1}^{n} v_{\alpha} a_{\alpha(l)}^{i j} D_{i j} v_{\bar{\alpha}(l)}+v_{\alpha} b^{i} v_{\alpha i}\right),
\end{gathered}
$$

where $\alpha(l)$ denotes the $l$-th element of multi-number $\alpha$, and $\bar{\alpha}(l)$ is the multi-number we get from $\alpha$ by leaving out its $l$-th element. Notice that

$$
2 v_{\alpha} a^{i j} v_{i j \alpha}=a^{i j}\left[\left|D^{n} v\right|^{2}\right]_{i j}-2 a^{i j} v_{i \alpha} v_{j \alpha}, \quad 2 v_{\alpha} b^{i} v_{i \alpha}=b^{i}\left(\left|D^{n} v\right|^{2}\right)_{i} .
$$

Hence integrating by parts and using Assumption 2.1, with $c_{p}=p(p-2) / 4 \geq 0$ we have

$$
\begin{gathered}
p\left|D^{n} v\right|^{p-2} \sum_{|\alpha|=n} v_{\alpha} a^{i j} v_{\alpha i j}=\frac{p}{2}\left|D^{n} v\right|^{p-2}\left(a^{i j}\left[\left|D^{n} v\right|^{2}\right]_{i j}-2 a^{i j} v_{i \alpha} v_{j \alpha}\right) \\
\sim-c_{p}\left|D^{n} v\right|^{p-4} a^{i j}\left[\left|D^{n} v\right|^{2}\right]_{i}\left[\left|D^{n} v\right|^{2}\right]_{j}-\frac{p}{2} a_{j}^{i j}\left|D^{n} v\right|^{p-2}\left[\left|D^{n} v\right|^{2}\right]_{i}-p\left|D^{n} v\right|^{p-2} a^{i j} v_{i \alpha} v_{j \alpha} \\
\preceq a_{j i}^{i j}\left|D^{n} v\right|^{p}-p\left|D^{n} v\right|^{p-2} a^{i j} v_{i \alpha} v_{j \alpha} \preceq-p\left|D^{n} v\right|^{p-2} a^{i j} v_{i \alpha} v_{j \alpha},
\end{gathered}
$$

and

$$
p\left|D^{n} v\right|^{p-2} \sum_{|\alpha|=n} v_{\alpha} b^{i} v_{i \alpha}=\frac{p}{2}\left|D^{n} v\right|^{p-2} b^{i}\left(\left|D^{n} v\right|^{2}\right)_{i}=b^{i}\left(\left|D^{n} v\right|^{p}\right)_{i} \sim-b_{i}^{i}\left|D^{n} v\right|^{p} \preceq 0 .
$$

Taking into account (4.4) and (4.5), from (4.2) we get

$$
A \preceq-p\left|D^{n} v\right|^{p-2} a^{i j} v_{i \alpha} v_{j \alpha}+B
$$

with

$$
B:=p\left|D^{n} v\right|^{p-2} \sum_{|\alpha|=n} v_{\alpha} \sum_{l=1}^{n} a_{\alpha(l)}^{i j} D_{i j} v_{\bar{\alpha}(l)} .
$$

Note that the computations in Eq. 4.4 are valid only for $p \geq 4$. For $p=2$ we can get (4.6) directly from Eq. 4.2 by noticing that by integrating by parts we have

$$
\begin{aligned}
2 \sum_{|\alpha|=n} v_{\alpha} a^{i j} v_{\alpha i j} & \sim-2 a^{i j} v_{\alpha i} v_{\alpha j}-2 a_{j}^{i j} v_{\alpha} v_{\alpha i}=-2 a^{i j} v_{\alpha i} v_{\alpha j}-a_{j}^{i j}\left[\left|D^{n} v\right|^{2}\right]_{i} \\
& \sim-2 a^{i j} v_{\alpha i} v_{\alpha j}+a_{j i}^{i j}\left|D^{n} v\right|^{2} \preceq-2 a^{i j} v_{\alpha i} v_{\alpha j} .
\end{aligned}
$$

For $p \in(2,4)$ we modify the calculations in Eq. 4.4 by taking $\phi_{\varepsilon}\left(\left|D^{n} v\right|^{2}\right)$ in place of $\left|D^{n} v\right|^{p-2}$ in Eq. 4.3, where $\phi_{\varepsilon}$ for each $\varepsilon>0$ is a function defined by

$$
\phi_{\varepsilon}(r)=\int_{0}^{|r|} \varphi_{\varepsilon}(s) d s, \quad r \in \mathbb{R}
$$

with a continuous function $\varphi_{\varepsilon}$ on $[0, \infty)$, supported on $[\varepsilon, \infty)$ such that $0 \leq \varphi_{\varepsilon}(r) \leq(p-2) r^{(p-4) / 2} / 2, \quad$ for $r \geq 0, \quad \varphi_{\varepsilon}(r)=(p-2) r^{(p-4) / 2} / 2 \quad$ for $r \geq 2 \varepsilon$. 
Clearly, $\phi_{\varepsilon}$ is continuously differentiable such

$$
\phi_{\varepsilon}^{\prime}(r)=d \phi_{\varepsilon}(r) / d r \geq 0 \text { for } r \geq 0,
$$

$\lim _{\varepsilon \rightarrow 0} \phi_{\varepsilon}(r)=|r|^{(p-2) / 2}, \quad\left|\phi_{\varepsilon}(r)\right| \leq N|r|^{(p-2) / 2}, \quad\left|\phi_{\varepsilon}^{\prime}(r)\right| \leq N|r|^{(p-4) / 2} \quad$ for $r \in \mathbb{R}$, and

$$
\Phi_{\varepsilon}(r):=\int_{0}^{r} \phi_{\varepsilon}(s) d s \leq N r^{p / 2} \text { for } r \geq 0
$$

with a constant $N$ depending only on $p$. Then noticing that

$$
\begin{gathered}
\phi_{\varepsilon}\left(\left|D^{n} v\right|^{2}\right) a^{i j}\left[\left|D^{n} v\right|^{2}\right]_{i j} \sim-\phi_{\varepsilon}^{\prime}\left(\left|D^{n} v\right|^{2}\right) a^{i j}\left[\left|D^{n} v\right|^{2}\right]_{i}\left[\left|D^{n} v\right|^{2}\right]_{j}-\phi_{\varepsilon}\left(\left|D^{n} v\right|^{2}\right) a_{j}^{i j}\left[\left|D^{n} v\right|^{2}\right]_{i} \\
\leq-\phi_{\varepsilon}\left(\left|D^{n} v\right|^{2}\right) a_{j}^{i j}\left[\left|D^{n} v\right|^{2}\right]_{i},
\end{gathered}
$$

we get

$$
\begin{aligned}
p \phi_{\varepsilon}\left(\left|D^{n} v\right|^{2}\right) \sum_{|\alpha|=n} v_{\alpha} a^{i j} v_{\alpha i j} & =\frac{p}{2} \phi_{\varepsilon}\left(\left|D^{n} v\right|^{2}\right)\left(a^{i j}\left[\left|D^{n} v\right|^{2}\right]_{i j}-2 a^{i j} v_{i \alpha} v_{j \alpha}\right) \\
& \preceq-\frac{p}{2} a_{j}^{i j} \phi_{\varepsilon}\left(\left|D^{n} v\right|^{2}\right)\left[\left|D^{n} v\right|^{2}\right]_{i}-p \phi_{\varepsilon}\left(\left|D^{n} v\right|^{2}\right) a^{i j} v_{i \alpha} v_{j \alpha} \\
& =-\frac{p}{2} a_{j}^{i j} D_{i} \Phi_{\varepsilon}\left(\left|D^{n} v\right|^{2}\right)-p \phi_{\varepsilon}\left(\left|D^{n} v\right|^{2}\right) a^{i j} v_{i \alpha} v_{j \alpha} \\
& \preceq a_{j i}^{i j} \Phi_{\varepsilon}\left(\left|D^{n} v\right|^{2}\right)-p \phi_{\varepsilon}\left(\left|D^{n} v\right|^{2}\right) a^{i j} v_{i \alpha} v_{j \alpha} \\
& \preceq-p \phi_{\varepsilon}\left(\left|D^{n} v\right|^{2}\right) a^{i j} v_{i \alpha} v_{j \alpha} .
\end{aligned}
$$

Consequently,

$$
p \phi_{\varepsilon}\left(\left|D^{n} v\right|^{2}\right) \sum_{|\alpha|=n} v_{\alpha} a^{i j} v_{\alpha i j} \preceq-p \phi_{\varepsilon}\left(\left|D^{n} v\right|^{2}\right) a^{i j} v_{i \alpha} v_{j \alpha} \quad \text { for } \varepsilon>0 .
$$

Letting here $\varepsilon \rightarrow 0$, by Lebesgue's theorem on dominated convergence we obtain

$$
p\left|D^{n} v\right|^{p-2} \sum_{|\alpha|=n} v_{\alpha} a^{i j} v_{\alpha i j} \preceq-p\left|D^{n} v\right|^{p-2} a^{i j} v_{i \alpha} v_{j \alpha} .
$$

Hence, taking into account (4.5), from Eq. 4.2 we get (4.6) also for $p \in(2,4)$, i.e., (4.6) is valid for all $p \geq 2$. We estimate $B$ by using the simple inequality

$$
\left|v_{\alpha} \sum_{l=1}^{n} a_{\alpha(l)}^{i j} D_{i j} v_{\bar{\alpha}(l)}\right| \leq \varepsilon^{-1}\left|v_{\alpha}\right|^{2}+\varepsilon n \sum_{l=1}^{n}\left|a_{\alpha(l)}^{i j} D_{i j} v_{\bar{\alpha}(l)}\right|^{2}
$$

for every $\varepsilon>0$ and multi-number $\alpha$, to get

$$
B \leq p \varepsilon^{-1}\left|D^{n} v\right|^{p}+\varepsilon n p\left|D^{n} v\right|^{p-2} C \quad \text { with } \quad C:=\sum_{|\alpha|=n} \sum_{l=1}^{n}\left|a_{\alpha(l)}^{i j} D_{i j} v_{\bar{\alpha}(l)}\right|^{2} .
$$

Using estimate Eq. 3.24 with $V^{i j}:=D_{i j} v_{\bar{\alpha}(l)}$ for each $l=1,2, \ldots, n$ and multi-number $\alpha$ of length $n$, we get

$$
C \leq N \sum_{|\alpha|=n} \sum_{l=1}^{n} a^{i j} D_{i k} v_{\bar{\alpha}(l)} D_{j k} v_{\bar{\alpha}(l)} \leq N^{\prime} \sum_{|\alpha|=n} a^{i j} D_{i} v_{\alpha} D_{j} v_{\alpha}
$$

with a constant $N^{\prime}=N^{\prime}(d, K, n)$. Thus, choosing $\varepsilon$ sufficiently small in the inequality in Eq. 4.7, from Eq. 4.6 we obtain $A \preceq 0$, which proves the lemma. 
For the following lemmas recall the definition of the operators $I^{\xi}, I^{\eta}$ and $J^{\eta}$ by Eqs. 3.10 and 3.11, and notice that the identities

$$
\begin{aligned}
& 2 v I^{\xi} v=I^{\xi} v^{2}-\left(I^{\xi} v\right)^{2} \\
& 2 v J^{\eta} v=J^{\eta} v^{2}-\left(I^{\eta} v\right)^{2}
\end{aligned}
$$

hold for $(t, x, z) \in H_{T} \times Z$, for functions $v=v(x)$ of $x \in \mathbb{R}^{d}$.

Lemma 4.3 Let Assumption 2.3 hold with an integer $m \geq 0$. Assume $p=2^{k}$ for an integer $k \geq 1$. Then for $n=0,1, \ldots, m$

$$
\mathcal{I}(v):=\sum_{|\alpha|=n}\left(\left|D^{n} v\right|^{p-2} D_{\alpha} v, D_{\alpha} I^{\xi} v\right) \leq N \bar{\xi}|v|_{W_{p}^{n}}^{p}
$$

with a constant $N=N(d, p, m, K)$ for all $v \in W_{p}^{n+1}$ and $(t, z) \in[0, T] \times Z$.

Proof Consider first the case $n=0$. Then by identity (4.8)

$$
\begin{aligned}
& |v|^{p-2} v I^{\xi} v=\frac{1}{2}|v|^{p-2} I^{\xi} v^{2}-\frac{1}{2}|v|^{p-2}\left(I^{\xi} v\right)^{2}=\frac{1}{2}|v|^{p-4} v^{2} I^{\xi} v^{2}-\frac{1}{2}|v|^{p-2}\left(I^{\xi} v\right)^{2} \\
& =\frac{1}{4}|v|^{p-4} I^{\xi} v^{4}-\frac{1}{2}|v|^{p-2}\left(I^{\xi} v\right)^{2}-\frac{1}{4}|v|^{p-4}\left(I^{\xi} v^{2}\right)^{2}=\cdots=\frac{1}{p} I^{\xi} v^{p}-A
\end{aligned}
$$

with

$$
A=\sum_{j=1}^{k} 2^{-j}|v|^{p-2^{j}}\left(I^{\xi} v^{j}\right)^{2} \geq 0 .
$$

Hence integrating over $\mathbb{R}^{d}$, by Eq. 3.18 we have

$$
\mathcal{I}(v) \leq \frac{1}{p} \int_{\mathbb{R}^{d}} I^{\xi} v^{p} d x \leq N \bar{\xi}|v|_{L_{p}}^{p} .
$$

Assume now that $n \geq 1$ and let $\alpha$ be a multi-number of length $n$. Then

$$
\left(I^{\xi} v\right)_{k}=I^{\xi} v_{k}+\xi_{k}^{i} T^{\xi} v_{i}, \quad\left(T^{\xi} v\right)_{k}=T^{\xi} v_{k}+\xi_{k}^{i} T^{\xi} v_{i}
$$

for $k=1,2, \ldots, d$. (Recall that we use the notation $g_{\alpha}=D_{\alpha} g$ for multi-numbers $\alpha$.) Hence, by induction on the length $n$ of the multi-number of $\alpha$, we obtain

$$
\left(I^{\xi} v\right)_{\alpha}=I^{\xi} v_{\alpha}+\sum_{1 \leq|\beta| \leq n} q^{\alpha, \beta} T^{\xi} v_{\beta}
$$

with some polynomial $q^{\alpha, \beta}$ of $\left\{\xi_{\gamma}^{i}: 1 \leq|\gamma| \leq n, i=1, \ldots, d\right\}$ for each multi-number $\beta$ of length between 1 and $n$. The degree of these polynomials is not greater than $n$, their constant term is zero, and the other coefficients are nonnegative integers. Hence

$$
\left|D^{n} v\right|^{p-2} v_{\alpha}\left(I^{\xi} v\right)_{\alpha}=\left|D^{n} v\right|^{p-2} v_{\alpha} I^{\xi} v_{\alpha}+\sum_{1 \leq|\beta| \leq n}\left|D^{n} v\right|^{p-2} v_{\alpha} q^{\alpha, \beta} T^{\xi} v_{\beta},
$$

where the repeated multi-numbers $\alpha$ mean summation over $|\alpha|=n$. By using the same calculation as in Eq. 4.11 we have

$$
\begin{gathered}
\left|D^{n} v\right|^{p-2} v_{\alpha} I^{\xi} v_{\alpha}=\frac{1}{2}\left|D^{n} v\right|^{p-2}\left\{I^{\xi}\left(\left|D^{n} v\right|^{2}\right)-\sum_{|\alpha|=n}\left(I^{\xi} v_{\alpha}\right)^{2}\right\} \\
\quad \leq \frac{1}{2}\left|D^{n} v\right|^{p-4}\left|D^{n} v\right|^{2} I^{\xi}\left(\left|D^{n} v\right|^{2}\right) \leq \cdots \leq \frac{1}{p} I^{\xi}\left(\left|D^{n} v\right|^{p}\right) .
\end{gathered}
$$

Thus

$$
\left|D^{n} v\right|^{p-2} v_{\alpha}\left(I^{\xi} v\right)_{\alpha} \leq \frac{1}{p} I^{\xi}\left(\left|D^{n} v\right|^{p}\right)+N \bar{\xi}\left|D^{n} v\right|^{p-1} \sum_{1 \leq|\beta| \leq n}\left|T^{\xi} v_{\beta}\right|
$$




$$
\leq \frac{1}{p} I^{\xi}\left(\left|D^{n} v\right|^{p}\right)+N \bar{\xi}\left|D^{n} v\right|^{p}+N^{\prime} \bar{\xi} \sum_{1 \leq|\beta| \leq n}\left|T^{\xi} v_{\beta}\right|^{p}
$$

with constants $N$ and $N^{\prime}$ depending only on $m, d, p$ and $K$. Integrating here over $\mathbb{R}^{d}$ we get (4.10).

Lemma 4.4 Let Assumption 2.2 hold with an integer $m \geq 0$. Assume $p=2^{k}$ for an integer $k \geq 1$. Then for $n=0,1, \ldots, m$ we have

$$
\mathfrak{J}(v):=\sum_{|\alpha|=n}\left(\left|D^{n} v\right|^{p-2} D_{\alpha} v, D_{\alpha} J^{\eta} v\right) \leq N \bar{\eta}^{2}|v|_{W_{p}^{n}}^{p}
$$

with a constant $N=N(d, p, m, K)$ for all $v \in W_{p}^{n+2}$ and $(t, z) \in[0, T] \times Z$.

Proof Consider first the case $n=0$. Then using identity (4.9) and proceeding with the proof in the same way as in the proof of the previous lemma we get

$$
v^{p-2} v J^{\eta} v=\frac{1}{2} v^{p-2} J^{\eta}\left(v^{2}\right)-\frac{1}{2} v^{p-2}\left(I^{\eta} v\right)^{2}=\cdots=\frac{1}{p} J^{\eta} v^{p}-B
$$

with

$$
B=\sum_{j=1}^{k} 2^{-j}|v|^{p-2^{j}}\left(I^{\eta} v^{j}\right)^{2} \geq 0 .
$$

Integrating here over $\mathbb{R}^{d}$ by Eq. 3.19 we have

$$
\mathfrak{J}(v) \leq \frac{1}{p} \int_{\mathbb{R}^{d}} J^{\eta} v^{p} d x \leq N \bar{\eta}^{2}|v|_{L_{p}}^{p} .
$$

Assume now that $n \geq 1$ and let $\alpha$ be a multi-number of length $n$. Then for $\left(T^{\eta} v\right)_{k}:=$ $D_{k}\left(T^{\eta} v\right),\left(I^{\eta} v\right)_{k}:=D_{k}\left(I^{\eta} v\right)$ and $\left(J^{\eta} v\right)_{k}:=D_{k}\left(J^{\eta} v\right)$ we have

$$
\left(T^{\eta} v\right)_{k}=T^{\eta} v_{k}+\eta_{k}^{i} T^{\eta} v_{i}, \quad\left(I^{\eta} v\right)_{k}=I^{\eta} v_{k}+\eta_{k}^{i} T^{\eta} v_{i}, \quad\left(J^{\eta} v\right)_{k}=J^{\eta} v_{k}+\eta_{k}^{i} I^{\eta} v_{i}
$$

for every $k=1, \ldots, d$. Hence by induction on the length of $\alpha$ we get

$$
\left(J^{\eta} v\right)_{\alpha}=J^{\eta} v_{\alpha}+\sum_{1 \leq|\beta| \leq n} p^{\alpha, \beta} I^{\eta} v_{\beta}+\sum_{1 \leq|\beta| \leq n} q^{\alpha, \beta} T^{\eta} v_{\beta},
$$

with some polynomials $p^{\alpha, \beta}$ and $q^{\alpha, \beta}$ of $\left\{\eta_{\gamma}^{i}: 1 \leq|\gamma| \leq n, i=1, \ldots, d\right\}$. The degree of these polynomials is not greater than $n$, their constant term is zero, the coefficients of each first order term in the polynomials $q^{\alpha, \beta}$ is also zero, all the other coefficients in $p^{\alpha, \beta}$ and $q^{\alpha, \beta}$ are nonnegative integers. Hence we get

$$
\left|D^{n} v\right|^{p-2} v_{\alpha}\left(J^{\eta} v\right)_{\alpha}=\left|D^{n} v\right|^{p-2} v_{\alpha} J^{\eta} v_{\alpha}+A^{\beta}+B^{\beta}
$$

with

$$
A^{\beta}:=\left|D^{n} v\right|^{p-2} v_{\alpha} p^{\alpha, \beta} I^{\eta} v_{\beta}, \quad B^{\beta}:=\left|D^{n} v\right|^{p-2} v_{\alpha} q^{\alpha, \beta} T^{\eta} v_{\beta},
$$

where repeated $\alpha$ means summation over the multi-numbers $\alpha$ of length $n$.

Clearly, for all $\beta$ we have

$$
\left|B^{\beta}\right| \leq N \bar{\eta}^{2}|D v|^{p-1}\left|T^{\eta} v_{\beta}\right|
$$

with constants $N=N(m, K, d)$. For $|\beta| \leq n-1$ we estimate $A^{\beta}$ in the same way to get

$$
\left|A^{\beta}\right| \leq N \bar{\eta}\left|D^{n} v\right|^{p-1}\left|I^{\eta} v_{\beta}\right|
$$


and for $|\beta|=n$ we use Young's inequality to write

$$
\begin{gathered}
\left|v_{\alpha} p^{\alpha, \beta} I^{\eta} v_{\beta}\right| \leq \varepsilon\left|I^{\eta} v_{\beta}\right|^{2}+\varepsilon^{-1}\left|v_{\alpha} p^{\alpha, \beta}\right|^{2} \leq \varepsilon\left|I^{\eta} v_{\beta}\right|^{2}+\varepsilon^{-1}\left|D^{n} v\right|^{2} \sum_{|\alpha|=n}\left|p^{\alpha, \beta}\right|^{2} \\
\leq \varepsilon\left|I^{\eta} v_{\beta}\right|^{2}+N \varepsilon^{-1} \bar{\eta}^{2}\left|D^{n} v\right|^{2} .
\end{gathered}
$$

Hence for $|\beta|=n$ we have

$$
\left|A^{\beta}\right| \leq \varepsilon\left|D^{n} v\right|^{p-2}\left|I^{\eta} v_{\beta}\right|^{2}+N \varepsilon^{-1} \bar{\eta}^{2}\left|D^{n} v\right|^{p}
$$

for $\varepsilon>0$ with a constant $N=N(K, d, m)$. Calculating as in Eq. 4.14 we obtain

$$
\begin{gathered}
\left|D^{n} v\right|^{p-2} v_{\alpha} J^{\eta} v_{\alpha} \leq \frac{1}{2}\left|D^{n} v\right|^{p-2}\left\{J^{\eta}\left(\left|D^{n} v\right|^{2}\right)-\sum_{|\alpha|=n}\left|I^{\eta} v_{\alpha}\right|^{2}\right\} \\
\leq \frac{1}{4}\left|D^{n} v\right|^{p-4}\left|D^{n} v\right|^{2} J^{\eta}\left(\left|D^{n} v\right|^{2}\right)-\frac{1}{2} \sum_{|\alpha|=n}\left|D^{n} v\right|^{p-2}\left|I^{\eta} v_{\alpha}\right|^{2} \leq \ldots \\
\leq \frac{1}{p} J^{\eta}\left(\left|D^{n} v\right|^{p}\right)-\frac{1}{2} \sum_{|\alpha|=n}\left|D^{n} v\right|^{p-2}\left|I^{\eta} v_{\alpha}\right|^{2} .
\end{gathered}
$$

Using these estimates, from Eq. 4.15 we obtain

$$
\begin{aligned}
& \left|D^{n} v\right|^{p-2} v_{\alpha}\left(J^{\eta} v\right)_{\alpha} \leq \frac{1}{p} J^{\eta}\left(\left|D^{n} v\right|^{p}\right)-\frac{1}{2} \sum_{|\alpha|=n}\left|D^{n} v\right|^{p-2}\left|I^{\eta} v_{\alpha}\right|^{2}+\varepsilon\left|D^{n} v\right|^{p-2} \sum_{|\beta|=n}\left|I^{\eta} v_{\beta}\right|^{2} \\
& \quad+N \varepsilon^{-1} \bar{\eta}^{2}\left|D^{n} v\right|^{p}+N \bar{\eta}^{2}\left|D^{n} v\right|^{p-1} \sum_{1 \leq|\beta| \leq n}\left|T^{\eta} v_{\beta}\right|+N \bar{\eta}\left|D^{n} v\right|^{p-1} \sum_{1 \leq|\beta| \leq n-1}\left|I^{\eta} v_{\beta}\right| .
\end{aligned}
$$

Choosing here $\varepsilon=1 / 2$, we get

$$
\begin{gathered}
\left|D^{n} v\right|^{p-2} v_{\alpha}\left(J^{\eta} v\right)_{\alpha} \leq \frac{1}{p} J^{\eta}\left(\left|D^{n} v\right|^{p}\right)+N \bar{\eta}^{2}|D v|^{p} \\
+N \bar{\eta}^{2}\left|D^{n} v\right|^{p-1} \sum_{1 \leq|\beta| \leq n}\left|T^{\eta} v_{\beta}\right|+N \bar{\eta}\left|D^{n} v\right|^{p-1} \sum_{1 \leq|\gamma| \leq n-1}\left|I^{\eta} v_{\gamma}\right| .
\end{gathered}
$$

By Hölder's inequality, taking into account (3.14) we have

$$
\begin{aligned}
& \int_{\mathbb{R}^{d}}\left|D^{n} v\right|^{p-1}\left|T^{\eta} v_{\beta}\right| d x \quad \leq N|v|_{W_{p}^{n}}^{p-1}\left|T^{\eta} v_{\beta}\right|_{L_{p}} \leq N^{\prime}|v|_{W_{p}^{n}}^{p} \quad \text { for }|\beta| \leq n, \\
& \int_{\mathbb{R}^{d}}\left|D^{n} v\right|^{p-1}\left|I^{\eta} v_{\gamma}\right| d x \leq N|v|_{W_{p}^{n}}^{p-1}\left|I^{\eta} v_{\gamma}\right|_{L_{p}} \leq N^{\prime} \bar{\eta}|v|_{W_{p}^{n}}^{p} \quad \text { for }|\gamma| \leq n-1
\end{aligned}
$$

with some constants $N=N(d, p)$ and $N^{\prime}=N^{\prime}(d, m, p, K)$. Integrating inequality (4.16) over $\mathbb{R}^{d}$ and using inequalities (3.19) and (4.17) we obtain (4.13).

Proof of Theorem 4.1 By the definition of $\mathcal{A}$ for $v \in W_{p}^{n+2}$ we have

$$
\sum_{|\alpha|=n}\left(\left|D^{n} v\right|^{p-2} D_{\alpha} v, D_{\alpha} \mathcal{A} v\right)=\sum_{|\alpha|=n}\left(\left|D^{n} v\right|^{p-2} D_{\alpha} v, D_{\alpha} \mathcal{L} v\right)+\int_{Z} \mathcal{I}(v) v(d z)+\int_{Z} \mathfrak{J}(v) \mu(d z),
$$

where $\mathcal{I}$ and $\mathfrak{J}$ are defined in Eqs. 4.10 and 4.13, respectively. Hence we get Theorem 4.1 by Lemmas $4.2,4.3$ and 4.4. 


\section{Proof of the Main Result}

\subsection{Uniqueness of the Generalised Solution}

Assume that Assumptions 2.1 through 2.4 hold with $m=0$. Let $u_{i} \in L_{p}\left([0, T], W_{p}^{1}\right)$ be generalised solutions to Eq. 1.1 with initial condition $u_{i}(0)=\psi$ for $i=1,2$. Then $v:=u_{1}-u_{2}$ is a generalised solution of Eq. 1.1 such that $v(0)=0$ and $f=0$. By Lemma 3.12 we have

$$
|v(t)|_{L_{p}}^{p}=\int_{0}^{t} Q\left(s, v_{s}\right)+Q^{\eta}\left(s, v_{s}\right)+Q^{\xi}\left(s, v_{s}\right)+p|v(s)|^{p-2} v(s) \mathcal{R} v(s) d s
$$

where

$$
\begin{aligned}
Q(s, v) & :=-\left(p(p-1)|v|^{p-2} D_{i} v, a^{i j}(s) D_{j} v\right)+\left(p|v|^{p-2} v, \bar{b}^{i}(s) D_{i} v+c v\right), \\
Q^{\eta}(s, v) & :=-\left(p(p-1)|v|^{p-2} D_{k} v, \mathcal{J}^{k} v\right)+\left(p|v|^{p-2} v, \mathcal{J}^{0} v\right), \\
Q^{\xi}(s, v) & :=\int_{Z}\left(p|v|^{p-2} v, I^{\xi} v\right) v(d z)
\end{aligned}
$$

for any $v \in W_{p}^{1}$, and recall that $\bar{b}^{i}=b^{i}-D_{j} a^{i j}$,

$$
I^{\xi} v=v\left(x+\xi_{s, z}(x)\right)-v(x)
$$

and $\mathcal{J}^{k}$ and $\mathcal{J}^{0}$ are defined in Eqs. 2.2 and 2.3.

We need the following lemma.

Lemma 5.1 For any $p \geq 2$ we have

$$
Q(s, v) \leq N|v|_{L_{p}}^{p}, \quad Q^{\eta}(s, v) \leq N_{1}|v|_{L_{p}}^{p}, \quad Q^{\xi}(s, v) \leq N_{2}|v|_{L_{p}}^{p}
$$

for all $v \in W_{p}^{1}$ and $s \in[0, T]$, with constants $N=N(d, p), N_{1}=N_{1}\left(d, p, K, K_{\bar{\eta}}\right)$, $N_{2}=N_{2}\left(d, p, K, K_{\bar{\xi}}\right)$.

Proof Notice that $|v|^{p}$ is a convex function of $v$. Hence we have

$$
I^{\xi}|v|^{p}-p|v|^{p-2} v I^{\xi} v \geq 0
$$

for all $(z, s, x) \in Z \times H_{T}$, which implies

$$
p|v|^{p-2} v I^{\xi} v=p|v|^{p-2} v I^{\xi} v-I^{\xi}|v|^{p}+I^{\xi}|v|^{p} \leq I^{\xi}|v|^{p} .
$$

Then by using estimate (3.18) in Lemma 3.11, we get the last estimate in Eq. 5.2. To prove the first and second estimates in Eq. 5.2 notice that

$$
Q(s, v)=p\left(|v|^{p-2} v, \mathcal{L}(s) v\right), \quad Q^{\eta}(s, v)=p\left(|v|^{p-2} v, \mathcal{M}(s) v\right)
$$

for $v \in W_{p}^{2}$. By Lemma 4.2, we obtain

$$
Q(s, v) \leq N|v|_{L_{p}}^{p} \quad \text { for } v \in W_{p}^{2} .
$$

Using the definition of the operators $J^{\eta}$ and $I^{\eta}$ and the convexity of the function $|v|^{p}$, we have

$$
p|v|^{p-2} v J^{\eta} v=p|v|^{p-2} v\left(I^{\eta} v-v_{i} \eta^{i}\right)=p|v|^{p-2} v I^{\eta} v+J^{\eta}|v|^{p}-I^{\eta}|v|^{p} \leq J^{\eta}|v|^{p},
$$


which by Eq. 3.19 in Lemma 3.11 implies

$$
Q^{\eta}(s, v) \leq N_{1}|v|_{L_{p}}^{p} \quad \text { for } v \in W_{p}^{2} .
$$

It remains to show $Q(s, v)$ and $Q^{\eta}(s, v)$ are continuous in $v \in W_{p}^{1}$. Let $\left\{v_{n}\right\}_{n=1}^{\infty}$ be a sequence of $W_{p}^{2}$ functions, which converges in the $W_{p}^{1}$ norm to some $v \in W_{p}^{1}$ as $n \rightarrow \infty$. We claim that $Q^{\eta}\left(s, v_{n}\right) \rightarrow Q^{\eta}(s, v)$. Clearly,

$$
Q^{\eta}\left(s, v_{n}\right)-Q^{\eta}(s, v)=p(p-1) B_{n}+p C_{n}
$$

with

$$
\begin{aligned}
B_{n} & :=\int_{\mathbb{R}^{d}}\left(|v|^{p-2} D_{k} v \mathcal{J}^{k} v-\left|v_{n}\right|^{p-2} D_{k} v_{n} \mathcal{J}^{k} v_{n}\right) d x, \\
C_{n} & :=\int_{\mathbb{R}^{d}}\left(\left|v_{n}\right|^{p-2} v_{n} \mathcal{J}^{0} v_{n}-|v|^{p-2} v \mathcal{J}^{0} v\right) d x .
\end{aligned}
$$

Observe that $B_{n}=B_{n}^{(1)}+B_{n}^{(2)}+B_{n}^{(3)}$ with

$$
\begin{aligned}
B_{n}^{(1)} & :=\int_{\mathbb{R}^{d}}\left(|v|^{p-2}-\left|v_{n}\right|^{p-2}\right) D_{k} v \mathcal{J}^{k} v d x, \\
B_{n}^{(2)} & :=\int_{\mathbb{R}^{d}}\left|v_{n}\right|^{p-2}\left(D_{k} v-D_{k} v_{n}\right) \mathcal{J}^{k} v d x, \\
B_{n}^{(3)} & :=\int_{\mathbb{R}^{d}}\left|v_{n}\right|^{p-2} D_{k} v_{n}\left(\mathcal{J}^{k} v-\mathcal{J}^{k} v_{n}\right) d x .
\end{aligned}
$$

By Hölder's inequality,

$$
\begin{aligned}
& \left|B_{n}^{(1)}\right| \leq\left.|| v_{n}\right|^{p-2}-\left.|v|^{p-2}\right|_{L^{\frac{p}{p-2}}}\left|D_{k} v\right|_{L^{p}}\left|\mathcal{J}^{k} v\right|_{L^{p}}, \\
& \left|B_{n}^{(2)}\right| \leq\left|v_{n}\right|_{L^{p}}^{p-2}\left|D_{k} v-D_{k} v_{n}\right|_{L^{p}}\left|\mathcal{J}^{k} v\right|_{L^{p}} \\
& \left|B_{n}^{(3)}\right| \leq\left|v_{n}\right|_{L_{p}}^{p-2}\left|D_{k} v_{n}\right|_{L_{p}}\left|\mathcal{J}^{k} v-\mathcal{J}^{k} v_{n}\right|_{L_{p}} .
\end{aligned}
$$

Since $v_{n} \rightarrow v$ in $W_{p}^{1}$, it is easy to see that $B_{n}^{(i)} \rightarrow 0$ for $i=2,3$. By Lemma 3.14 we have

$$
\left.|| v_{n}\right|^{p-2}-\left.|v|^{p-2}\right|_{L} \frac{p}{p-2} \rightarrow 0
$$

which gives $\lim _{n \rightarrow \infty} B_{n}^{(1)}=0$. We get in the same way that $\lim _{n \rightarrow \infty} C_{n}=0$. The continuity of $Q(s, v)$ in $v \in W_{p}^{1}$ can be proved similarly.

Using the above lemma and Assumption 2.4, from Eq. 5.1 we get

$$
|v(t)|_{L_{p}}^{p} \leq N \int_{0}^{t}|v(s)|_{L_{p}}^{p} d s \quad t \in[0, T]
$$

with a constant $N$, which completes the proof of the uniqueness.

\subsection{Existence of a Generalised Solution}

In the whole subsection we assume that Assumptions 2.1 through2.5 hold with given real numbers $m \geq 1$ and $p \geq 2$. We prove the existence of a solution to Eq. 1.1 with initial condition $u(0)=\psi$ in several steps below. In the first two steps, we make the additional assumptions that $\mathcal{R}=0, p=2^{k}$ for some integer $k \geq 1$ and that $m$ is an integer. We construct a solution $u$ in $L_{p}\left([0, T], W_{p}^{m}\right)$ by approximation procedures, and estimate its norm in $L_{p}\left([0, T], W_{p}^{s}\right)$ for integers $s=0,1, \ldots, m$ by the right-hand side of Eq. 2.6. Hence, using standard results from interpolation theory we prove the existence of a generalised 
solution $u \in L_{p}\left([0, T], V_{p}^{m}\right)$ without assuming that $p=2^{k}$. Then using the method of continuity(see e.g., [21]) we extend this result to equations with $\mathcal{R}$ satisfying Assumption 2.4. Hence using interpolation again, we dispense with the assumption that $m$ is an integer. Moreover, we prove that $u \in C\left([0, T], V_{p}^{s}\right) \cap C_{w}\left([0, T], V_{p}^{m}\right)$ for every $s<m$, and obtain also the estimate Eq. 2.6. We note that similar interpolation arguments are used in [14] to obtain estimates in $L_{p}$-spaces for solutions of stochastic finite difference schemes.

Step 1. First we assume that Assumptions 2.1, 2.2, 2.3 and 2.5 hold with integers $m \geq 1$ and $p=2^{k}$ for an integer $k \geq 1$. Moreover, we assume that $\psi$ and $f$ are compactly supported. Under these assumptions we approximate the Cauchy problem (1.1) with initial condition $u(0)=\psi$ by smoothing the data and the coefficients in the problem. Recall that for $\varepsilon>0$ and functions $v$ on $\mathbb{R}^{d}$ the notation $v^{(\varepsilon)}$ means the mollification $v^{(\varepsilon)}=S_{\varepsilon} v$ of $v$ defined in Eq. 3.5. We consider the Cauchy problem

$$
\begin{aligned}
d v(t, x) & =\left(\mathcal{A}_{\varepsilon}^{0}(t) v(t, x)+f^{(\varepsilon)}(t, x)\right) d t, \quad(t, x) \in H_{T}, \\
v(0, x) & =\psi^{(\varepsilon)}(x), \quad x \in \mathbb{R}^{d}
\end{aligned}
$$

for $\varepsilon \in\left(0, \varepsilon_{0}\right)$, where $\varepsilon_{0}$ is given in Corollary 3.6, and

$$
\mathcal{A}_{\varepsilon}^{0}:=\mathcal{L}_{\varepsilon}+\mathcal{M}_{\varepsilon}+\mathcal{N}_{\varepsilon}
$$

with operators $\mathcal{L}_{\varepsilon}, \mathcal{M}_{\varepsilon}$ and $\mathcal{N}_{\varepsilon}$, defined by

$$
\begin{gathered}
\mathcal{L}_{\varepsilon}=a_{\varepsilon}^{i j} D_{i j}+b^{(\varepsilon) i} D_{i}+c^{(\varepsilon)}, \quad a_{\varepsilon}=a^{(\varepsilon)}+\varepsilon \mathbb{I}, \\
\mathcal{M}_{\varepsilon} \varphi(x)=\int_{Z}\left\{\varphi\left(x+\eta_{t, z}^{(\varepsilon)}\right)-\varphi(x)-\eta_{t, z}^{(\varepsilon)} \nabla \varphi(x)\right\} \mu(d z), \\
\mathcal{N}_{\varepsilon} \varphi=\int_{Z}\left\{\varphi\left(x+\xi_{t, z}^{(\varepsilon)}\right)-\varphi(x)\right\} \nu(d z)
\end{gathered}
$$

for $\varphi \in C_{0}^{\infty}$. (Recall that II denotes the $d \times d$ unit matrix.)

Since $\psi^{(\varepsilon)}$ and $f^{(\varepsilon)}$ are compactly supported, they belong to $W_{2}^{n}$ for every $n \geq 0$. By standard results of the $L_{2}$-theory of parabolic PDEs, (5.4)-(5.5) has a unique solution $u_{\varepsilon}$, which is a continuous $W_{2}^{n}$-valued function of $t \in[0, T]$ for every $n \geq 0$ (see, e.g., [23] or [41]). Thus for any $\varphi \in \stackrel{C}{C}_{0}^{\infty}$ we have

$$
\begin{gathered}
\left(u_{\varepsilon}(t), \varphi\right)=\left(\psi^{(\varepsilon)}, \varphi\right) \\
+\int_{0}^{t}-\left(a_{\varepsilon}^{i j} D_{j} u_{\varepsilon}(s), D_{i} \varphi\right)+\left(\bar{b}^{i(\varepsilon)} D_{i} u_{\varepsilon}(s)+c^{(\varepsilon)} u_{\varepsilon}(s)+f^{(\varepsilon)}(s), \varphi\right) d s \\
+\int_{0}^{t}-\left(\mathcal{J}_{\varepsilon}^{i} u_{\varepsilon}(s), D_{i} \varphi\right)+\left(\mathcal{J}_{\varepsilon}^{0} u_{\varepsilon}(s), \varphi\right)+\left(\mathcal{N}_{\varepsilon} u_{\varepsilon}(s), \varphi\right) d s
\end{gathered}
$$

for $t \in[0, T]$, where $\mathcal{J}_{\varepsilon}^{i}$ and $\mathcal{J}_{\varepsilon}^{0}$ are defined as $\mathcal{J}^{i}$ and $\mathcal{J}^{0}$, respectively in Eq. 2.3, but with $\eta^{k(\varepsilon)}$ and $\eta_{k}^{l(\varepsilon)}$ in place of $\eta^{k}$ and $\eta_{k}^{l}$, respectively, for $k, l=1,2, \ldots, d$. Notice that Eq. 5.6 can be rewritten as

$$
\left(u_{\varepsilon}(t), \varphi\right)=\left(\psi^{(\varepsilon)}, \varphi\right)+\int_{0}^{t}\left(\mathcal{A}_{\varepsilon}^{0} u_{\varepsilon}(s)+f^{(\varepsilon)}(s), \varphi\right) d s, \quad t \in[0, T], \quad \varphi \in C_{0}^{\infty},
$$

and, equivalently, as

$$
\left(D_{\alpha} u_{\varepsilon}(t), \varphi\right)=\left(D_{\alpha} \psi^{(\varepsilon)}, \varphi\right)+\int_{0}^{t}\left(D_{\alpha} \mathcal{A}_{\varepsilon}^{0} u_{\varepsilon}(s)+D_{\alpha} f^{(\varepsilon)}(s), \varphi\right) d s \quad t \in[0, T], \quad \varphi \in C_{0}^{\infty}
$$

for all multi-numbers $\alpha$ of length $n$. By Sobolev embedding $u_{\varepsilon}$ is a continuous $W_{p}^{n}$-valued function for every $n \geq 0$ and $p \geq 2$. Hence by Lemma 3.13 we have

$$
\left|D^{n} u_{\varepsilon}\right|_{L_{p}}^{p}=\left|D^{n} \psi^{(\varepsilon)}\right|_{L_{p}}^{p}
$$




$$
+p \int_{0}^{t} \sum_{|\alpha|=n}\left(\left|D^{n} u_{\varepsilon}(s)\right|^{p-2} D_{\alpha} u_{\varepsilon}(s), D_{\alpha} \mathcal{A}_{\varepsilon}^{0} u_{\varepsilon}(s)+D_{\alpha} f^{(\varepsilon)}(s)\right) d s,
$$

which for $p=2^{k}$ by Theorem 4.1, known properties of mollifications and Young's inequality gives

$$
\begin{gathered}
\left|D^{n} u_{\varepsilon}\right|_{L_{p}}^{p} \leq\left|D^{n} \psi\right|_{L_{p}}^{p}+N \int_{0}^{t}\left|u_{\varepsilon}(s)\right|_{W_{p}^{n}}^{p}+\left(\left|D^{n} u_{\varepsilon}(s)\right|^{p-2} D_{\alpha} u_{\varepsilon}(s), D_{\alpha} f^{(\varepsilon)}(s)\right) d s \\
\leq\left|D^{n} \psi\right|_{L_{p}}^{p}+N \int_{0}^{t}\left\{\left|u_{\varepsilon}(s)\right|_{W_{p}^{n}}^{p}+\frac{p-1}{p}\left|u_{\varepsilon}(s)\right|_{W_{p}^{n}}^{p}+\frac{1}{p}|f(s)|_{W_{p}^{n}}^{p}\right\} d s .
\end{gathered}
$$

This via Gronwall's lemma implies that for $\varepsilon \in\left(0, \varepsilon_{0}\right)$

$$
\sup _{t \in[0, T]}\left|u_{\varepsilon}(t)\right|_{W_{p}^{n}}^{p} \leq N\left(|\psi|_{W_{p}^{n}}^{p}+\int_{0}^{T}|f(t)|_{W_{p}^{n}}^{p} d t\right)
$$

for $n=0,1, \ldots, m$ with a constant $N=N\left(T, p, d, m, K, K_{\bar{\xi}}, K_{\bar{\eta}}\right)$. For $r>1$ and $p \geq 2$ we denote by $\mathbb{W}_{p, r}^{n}$ the space of $W_{p}^{n}$-valued Borel functions $v=v(t)$ of $t \in[0, T]$ such that

$$
|v|_{\mathbb{W}_{p, r}^{n}}:=\left(\int_{0}^{T}|v(t)|_{W_{p}^{n}}^{r} d t\right)^{1 / r}<\infty
$$

We use also the notation $\mathbb{W}_{p}^{n}$ and $\mathbb{L}_{p}$ for $\mathbb{W}_{p, p}^{n}$ and $\mathbb{W}_{p, p}^{0}$, respectively. Observe that with this norm $\mathbb{W}_{p, r}^{n}$ is a reflexive Banach space, and from Eq. 5.7 we have

$$
\left|u_{\varepsilon}\right|_{\mathbb{W}_{p, r}^{n}}^{p} \leq N\left(|\psi|_{W_{p}^{n}}^{p}+\int_{0}^{T}|f(t)|_{W_{p}^{n}}^{p} d t\right)
$$

for all $\varepsilon \in\left(0, \varepsilon_{0}\right), p=2^{k}, r>1$ and $n=0,1,2, \ldots, m$, with a constant $N$ depending only on $T, p, d, m, K, K_{\bar{\xi}}$ and $K_{\bar{\eta}}$. Hence there exists a sequence of positive numbers $\left\{\varepsilon_{k}\right\}_{k \in \mathbb{N}}$ such that $\varepsilon_{k} \rightarrow 0$ for $k \rightarrow \infty$, and $u_{\varepsilon_{k}}$ converges weakly to a function $u$ in $\mathbb{W}_{p, r}^{n}$ for every $n=0,1, \ldots, m$ and integers $r>1$. Letting $\varepsilon=\varepsilon_{k} \rightarrow 0$ in Eq. 5.8, we get

$$
|u|_{\mathbb{W}_{p, r}^{n}}^{p} \leq N\left(|\psi|_{W_{p}^{n}}^{p}+\int_{0}^{T}|f(t)|_{W_{p}^{n}}^{p} d t\right) .
$$

Our aim now is to pass to the limit in Eq. 5.6 along $\varepsilon_{k} \rightarrow 0$. To this end we take a realvalued bounded Borel function $h$ of $t \in[0, T]$, multiply both sides of Eq. 5.6 with $h(t)$ and then integrate it against $d t$ over [0,T]. Thus for a fixed $\varphi \in C_{0}^{\infty}$ and taking $\varepsilon_{k}$ in place of $\varepsilon$, we obtain

$$
F\left(u_{\varepsilon_{k}}\right)=\int_{0}^{T}\left(\psi^{\left(\varepsilon_{k}\right)}, \varphi\right) h(t) d t+\sum_{i=1}^{4} F_{k}^{i}\left(u_{\varepsilon_{k}}\right)+\int_{0}^{T} \int_{0}^{t}\left(f^{\left(\varepsilon_{k}\right)}(s), \varphi\right) h(t) d s d t,
$$

where $F$ and $F_{k}^{i}$ are functionals defined for $v \in \mathbb{W}_{p}^{1}$ by

$$
\begin{gathered}
F(v)=\int_{0}^{T}(v(t), \varphi) h(t) d t \\
F_{k}^{1}(v)=\int_{0}^{T} h(t) \int_{0}^{t}-\left(a_{\varepsilon_{k}}^{i j} D_{j} v(s), D_{i} \varphi\right)+\left(\bar{b}^{i\left(\varepsilon_{k}\right)} D_{i} v(s)+c^{\left(\varepsilon_{k}\right)} v(s), \varphi\right) d s d t \\
F_{k}^{2}(v)=-\int_{0}^{T} h(t) \int_{0}^{t}\left(\mathcal{J}_{\varepsilon_{k}}^{i} v(s), D_{i} \varphi\right) d s d t \\
F_{k}^{3}(v)=\int_{0}^{T} h(t) \int_{0}^{t}\left(\mathcal{J}_{\varepsilon_{k}}^{0} v(s), \varphi\right) d s d t
\end{gathered}
$$




$$
F_{k}^{4}(v)=\int_{0}^{T} h(t) \int_{0}^{t}\left(\mathcal{N}_{\varepsilon_{k}} v(s), \varphi\right) d s d t .
$$

For each $i$ define also the functional $F^{i}$ in the same way as $F_{k}^{i}$ is defined above, but with $a$, $b, c, \mathcal{J}^{i}, \mathcal{J}^{0}$ and $\mathcal{N}$ in place of $a_{\varepsilon_{k}}, b^{\left(\varepsilon_{k}\right)}, c^{\left(\varepsilon_{k}\right)}, \mathcal{J}_{\varepsilon_{k}}^{i}, \mathcal{J}_{\varepsilon_{k}}^{0}$ and $\mathcal{N}_{\varepsilon_{k}}$, respectively. Clearly, due to the boundedness of $h$ we have a constant $C$ such that for all $v \in \mathbb{W}_{p}^{1}$

$$
F(v) \leq C|v|_{\mathbb{L}_{p}}|\varphi|_{L_{q}} \leq C|v|_{\mathbb{W}_{p}^{1}}|\varphi|_{L_{q}},
$$

where $q=p /(p-1)$. This means $F \in \mathbb{W}_{p}^{1 *}$, the Banach space of bounded linear functionals on $\mathbb{W}_{p}^{1}$. To take the limit $k \rightarrow \infty$ in Eq. 5.10 we show below that $F_{k}^{i}$ and $F^{i}$ are in $\mathbb{W}_{p}^{1 *}$, and $F_{k}^{i} \rightarrow F^{i}$ strongly in $\mathbb{W}_{p}^{1 *}$, for every $i$ as $k \rightarrow \infty$.

Lemma 5.2 The functionals $F^{i}$ and $F_{k}^{i}$ belong to $\mathbb{W}_{p}^{1 *}$ for $i=1,2,3,4$, for sufficiently large $k$.

Proof Since the functions $h, a_{\varepsilon}, \bar{b}^{(\varepsilon)}$ and $c^{(\varepsilon)}$ are in magnitude bounded by a constant, by Hölder's inequality we have

$$
\left|F_{k}^{1}(v)\right| \leq N|v|_{\mathbb{W}_{p}^{1}}|\varphi|_{W_{q}^{1}}
$$

with a constant $N$ independent of $v$, which shows that $F_{k}^{1} \in \mathbb{W}_{p}^{1 *}$ for all $k$. In the same way we get $F^{1} \in \mathbb{W}_{p}^{1 *}$. Using that by Taylor's formula

$$
v\left(x+\theta \eta^{(\varepsilon)}\right)-v(x)=\int_{0}^{1} D_{i} v\left(x+\vartheta \theta \eta^{(\varepsilon)}\right) \theta \eta^{(\varepsilon) i} d \vartheta
$$

and taking into account that $|h|$ is bounded by a constant, we have

$$
\left|F_{k}^{2}(v)\right| \leq C \int_{0}^{1} \int_{0}^{1} \int_{0}^{T} \int_{Z} \int_{\mathbb{R}^{d}}\left|D v\left(s, x+\vartheta \theta \eta_{s, z}^{\left(\varepsilon_{k}\right)}(x)\right)\right| \bar{\eta}^{2}(z)|D \varphi(x)| d x \mu(d z) d s d \theta d \vartheta .
$$

Hence by Hölder's inequality and then the change of variable $y=x+\vartheta \theta \eta_{s, z}^{\left(\varepsilon_{k}\right)}(x)$, by Corollary 3.6 we get a constant $C$ such that for sufficiently large $k$

$$
\left|F_{k}^{2}(v)\right| \leq C \int_{Z} \bar{\eta}^{2}(z) \mu(d z)|v|_{\mathbb{W}_{p}^{1}}|D \varphi|_{L_{q}}=C K_{\eta}^{2}|v|_{\mathbb{W}_{p}^{1}}|D \varphi|_{L_{q}},
$$

which proves that $F^{2} \in \mathbb{W}_{p}^{1 *}$ for sufficiently large $k$. We can prove in the same way that $F^{2}, F_{k}^{i} \in \mathbb{W}_{p}^{1 *}$ and $F^{i} \in \mathbb{W}_{p}^{1 *}$ for $i=3,4$, for sufficiently large $k$.

Lemma 5.3 For every $i=1,2,3,4$

$$
\lim _{k \rightarrow \infty} \sup _{|v|_{\mathbb{W}_{p}^{1}} \leq 1}\left|\left(F_{k}^{i}-F^{i}\right)(v)\right|=0 .
$$

Proof Since $h$ is bounded, for a constant $N$ we have

$$
\left|F_{k}^{1}(v)-F^{1}(v)\right| \leq N \sum_{i=1}^{3} A_{k}^{i}(v)
$$


for all $k \geq 1$ with

$$
\begin{aligned}
A_{k}^{1}(v) & :=\int_{0}^{T} \int_{\mathbb{R}^{d}}\left|D_{j} v(s, x)\left\|a_{\varepsilon_{k}}^{i j}(s, x)-a^{i j}(s, x)\right\| D_{i} \varphi(x)\right| d x d s, \\
A_{k}^{2}(v) & \left.:=\int_{0}^{T} \int_{\mathbb{R}^{d}}\left|v(s, x)\left\|\bar{b}^{i\left(\varepsilon_{k}\right)}(s, x)-\bar{b}^{i}(s, x)\right\| D_{i} \varphi(x)\right|\right) d x d s, \\
A_{k}^{3}(v) & :=\int_{0}^{T} \int_{\mathbb{R}^{d}}\left|v(s, x)\left\|c^{\left(\varepsilon_{k}\right)}(s, x)-c(s, x)\right\| \varphi(x)\right| d x d s .
\end{aligned}
$$

By Hölder's inequality

$$
\begin{gathered}
\sup _{|v|_{\mathbb{W}_{p}^{1}} \leq 1} A_{k}^{1}(v) \leq|| a_{\varepsilon_{k}}-\left.a|| D \varphi\right|_{\mathbb{L}_{q}}, \quad \sup _{|v|_{\mathbb{W}_{p}^{1}} \leq 1} A_{k}^{2}(v) \leq|| b^{\left(\varepsilon_{k}\right)}-b|| D \varphi||_{\mathbb{L}_{q}}, \\
\sup _{|v|_{\mathbb{W}_{p}^{1}} \leq 1} A_{k}^{3}(v) \leq\left|\left(c^{\left(\varepsilon_{k}\right)}-c\right) \varphi\right|_{\mathbb{L}_{q}},
\end{gathered}
$$

where $\mathbb{L}_{q}=\mathbb{W}_{q, q}^{0}$. Letting here $k \rightarrow \infty$ by Lebesgue's theorem on dominated convergence we get

$$
\lim _{k \rightarrow \infty} \sup _{|v|_{\mathbb{W}_{p}^{1}} \leq 1} A_{k}^{i}(v)=0 \quad \text { for } i=1,2,3,
$$

which gives (5.12) for $i=1$. Clearly,

$$
\left|F_{k}^{2}(v)-F^{2}(v)\right| \leq B_{k}^{1}(v)+B_{k}^{2}(v)
$$

with

$$
\begin{aligned}
B_{k}^{1}(v) & :=\int_{0}^{1} \int_{0}^{T} \int_{Z}\left(\left|\eta^{\left(\varepsilon_{k}\right)}-\eta\right|\left|v\left(s, \tau_{\theta \eta^{\left(\varepsilon_{k}\right)}}\right)-v(s)\right|,|D \varphi|\right) \mu(d z) d s d \theta \\
B_{k}^{2}(v) & :=\int_{0}^{1} \int_{0}^{T} \int_{Z}\left(\bar{\eta}\left|v\left(s, \tau_{\left.\theta \eta^{\left(\varepsilon_{k}\right.}\right)}\right)-v\left(s, \tau_{\theta \eta}\right)\right|,|D \varphi|\right) \mu(d z) d s d \theta
\end{aligned}
$$

Note that $\left|\eta^{(\varepsilon)}-\eta\right| \leq \varepsilon \bar{\eta}$ for all $t \in[0, T], x \in \mathbb{R}^{d}, z \in Z$ and $\varepsilon>0$. Moreover, by Taylor's formula, Minkowski's inequality and Corollary 3.6

$$
\left|v\left(s, \tau_{\theta \eta^{(\varepsilon)}}\right)-v(s)\right|_{L_{p}} \leq \bar{\eta} \int_{0}^{1}\left|D v\left(s, \tau_{\vartheta \theta \eta^{(\varepsilon)}}\right)\right|_{L_{p}} d \vartheta \leq N \bar{\eta}|D v(s)|_{L_{p}},
$$

$\left|v\left(s, \tau_{\theta \eta^{\left(\varepsilon_{k}\right)}}\right)-v\left(s, \tau_{\theta \eta}\right)\right|_{L_{p}} \leq \int_{0}^{1}\left\|\eta^{(\varepsilon)}-\eta\right\| D v\left(s,(1-\vartheta) \tau_{\theta \eta^{(\varepsilon)}}+\vartheta \tau_{\theta \eta}\right) \|_{L_{p}} d \vartheta \leq N \varepsilon \bar{\eta}|D v(s)|_{L_{p}}$ for $s \in[0, T], z \in Z$ and $\varepsilon \in\left(0, \varepsilon_{0}\right)$, with a constant $N=N(K, d, p)$. Hence by Hölder's inequality for sufficiently large $k$ we have

$$
B_{k}^{i} \leq \varepsilon_{k} N|v|_{\mathbb{W}_{p}^{1}}|D \varphi|_{L_{q}} \int_{Z} \bar{\eta}^{2}(z) \mu(d z)=\varepsilon_{k} N K_{\eta}^{2}|v|_{\mathbb{W}_{p}^{1}}|D \varphi|_{L_{q}} \quad \text { for } i=1,2,
$$

which by virtue of Eq. 5.13 proves (5.12) for $i=2$. We can prove similarly that Eq. 5.12 holds for $i=3,4$.

By the above lemmas, for $i=1,2,3,4$ we have $F_{k}^{i} \rightarrow F^{i}$ strongly in $\mathbb{W}_{p}^{1 *}$ as $k \rightarrow \infty$. Thus due to the convergence of $u_{\varepsilon_{k}}$ to $u$ weakly in $\mathbb{W}_{p}^{1}$, we have

$$
\lim _{k \rightarrow \infty} F\left(u_{\varepsilon_{k}}\right)=F(u), \quad \lim _{k \rightarrow \infty} F_{k}^{i}\left(u_{\varepsilon_{k}}\right)=F(u) \quad \text { for } i=1,2,3,4 .
$$


Clearly,

$$
\begin{aligned}
\lim _{k \rightarrow \infty} \int_{0}^{T}\left(\psi^{\left(\varepsilon_{k}\right)}, \varphi\right) d t & =\int_{0}^{T}(\psi, \varphi) d t \\
\lim _{k \rightarrow \infty} \int_{0}^{T} \int_{0}^{t}\left(f^{\left(\varepsilon_{k}\right)}(s), \varphi\right) d s d t & =\int_{0}^{T} \int_{0}^{t}(f(s), \varphi) d s d t .
\end{aligned}
$$

Thus taking $k \rightarrow \infty$ in Eq. 5.10 we obtain

$$
F(u)=\int_{0}^{T}(\psi, \varphi) h(t) d t+\sum_{i=1}^{4} F^{i}(u)+\int_{0}^{T} \int_{0}^{t}(f(s), \varphi) h(t) d s d t .
$$

This means for every bounded real function $h$ the function $u:[0, T] \rightarrow W_{p}^{1}$ satisfies the equation

$$
\int_{0}^{T} h(t)(u(t), \varphi) d t=\int_{0}^{T} h(t)(\psi, \varphi) d t+\int_{0}^{T} h(t) \int_{0}^{t}\langle\mathcal{A} u(s), \varphi\rangle+(f(s), \varphi) d s d t
$$

for every $\varphi \in C_{0}^{\infty}$. Thus for each $\varphi \in C_{0}^{\infty}$ (2.5) holds for $d t$-almost every $t \in[0, T]$. Hence taking into account that $u \in L_{p}\left([0, T], W_{p}^{1}\right)$, by Lemma $3.12 u$ has a modification, denoted also by $u$, which is continuous as an $L_{p}$-valued function and it is the solution of Eq. 1.1 with initial value $\psi$.

Step 2. Now we dispense with the additional assumption that $\psi$ and $f$ vanish for $|x| \geq R$ for some $R>0$. Let $\psi \in W_{p}^{m}$ and $f \in L_{p}\left([0, T], W_{p}^{m}\right)$ for $p=2^{k}$ for some integer $k \geq 1$. Then for integers $n \geq 1$ define $\psi^{n}$ and $f^{n}$ by

$$
\psi^{n}(x)=\psi(x) \chi_{n}(x), \quad f^{n}(t, x)=f(t, x) \chi_{n}(x), \quad t \in[0, T], x \in \mathbb{R}^{d},
$$

where $\chi_{n}(\cdot)=\chi(\cdot / n)$ with a nonnegative function $\chi \in C_{0}^{\infty}$, such that $\chi(x)=1$ for $|x| \leq 1$ and $\chi(x)=0$ for $|x| \geq 2$. Then by virtue of Step 2 (1.1) with $f^{n}$ in place of $f$ and with initial condition $u(0)=\psi^{n}$ has a solution $u^{n}$, i.e.,

$$
\begin{gathered}
\int_{0}^{T} h(t)\left(u^{n}(t), \varphi\right) d t=\int_{0}^{T} h(t)\left(\psi^{n}, \varphi\right) d t \\
+\int_{0}^{T} h(t) \int_{0}^{t}\left\langle\mathcal{A} u^{n}(s), \varphi\right\rangle+\left(f^{n}(s), \varphi\right) d s d t
\end{gathered}
$$

for every $\varphi \in C_{0}^{\infty}$ and bounded Borel function $h:[0, T] \rightarrow \mathbb{R}$. We also have estimate (5.9) with $u^{n}, \psi^{n}$ and $f^{n}$ in place of $u, \psi$ and $f$, respectively. Hence for any $n$ and $k$

$$
\left|u^{n}-u^{k}\right|_{\mathbb{W}_{p, r}^{j}}^{p} \leq N\left(\left|\psi^{n}-\psi^{k}\right|_{W_{p}^{j}}^{p}+\left|f^{n}-f^{k}\right|_{\mathbb{W}_{p}^{j}}^{p}\right)
$$

which shows that $u^{n}$ is a Cauchy sequence in $\mathbb{W}_{p, r}^{j}$, and hence it converges in the norm of $\mathbb{W}_{p, r}^{j}$ to some $u \in \mathbb{W}_{p, r}^{j}$ for every $j=0,1,2, \ldots, m$ and integers $r>1$. It is easy to pass to the limit in Eq. 5.15 and see that $u$ solves (1.1) with initial and free data $\psi$ and $f$. Clearly, $u$ satisfies also the estimate (5.9).

Set $\Psi_{p}^{m}:=H_{p}^{m}, \mathbb{F}_{p}^{m}:=L_{p}\left([0, T], H_{p}^{m}\right)$ and $\mathbb{U}_{p}^{m}:=L_{r}\left([0, T], H_{p}^{m}\right)$ for $m \in[1, \infty)$, $p \in[2, \infty)$ and for fixed $r>1$. Denote by $\mathbb{S}$ the operator that assigns the solution $u$ of Eq. 1.1 to $(\psi, f)$, the pair of initial and free data. By virtue of Step 2 we know that $\mathbb{S}$ is a continuous linear operator from $\Psi_{p}^{m} \times \mathbb{F}_{p}^{m}$ into $\mathbb{U}_{p}^{m}$ for $p=2^{k}$, with integers $k \geq 1$, for every integer $m \geq 1$, with operator norm, depending only on $p, d, T, m$ and on the constants $K$, $K_{\bar{\eta}}$ and $K_{\bar{\xi}}$. To show that this holds also for any $p \in[2, \infty)$ and any $m \in(1, \infty)$, we use some results from the theory of complex interpolation of Banach spaces. 
A pair of complex Banach spaces $A_{0}$ and $A_{1}$, which are continuously embedded into a Hausdorff topological vector space $\mathcal{H}$, is called an interpolation couple, and $\left[A_{0}, A_{1}\right]_{\theta}$ denotes the complex interpolation space between $A_{0}$ and $A_{1}$ with parameter $\theta \in(0,1)$. For an interpolation couple $A_{0}$ and $A_{1}$ the notation $A_{0}+A_{1}$ is used for subspace of vectors in $\mathcal{H},\left\{v_{0}+v_{1}: v_{0} \in A_{0}, v_{1} \in A_{1}\right\}$, equipped with the norm

$$
|v|_{A_{0}+A_{1}}:=\inf \left\{\left|v_{0}\right|_{A_{0}}+\left|v_{1}\right|_{A_{1}}: v=v_{0}+v_{1}, v_{0} \in A_{0}, v_{1} \in A_{1}\right\} .
$$

Then the following statements hold (see 1.9.3, 1.18.4 and 2.4.2 from [43]).

(i) If $A_{0}, A_{1}$ and $B_{0}, B_{1}$ are two interpolation couples and $S: A_{0}+A_{1} \rightarrow B_{0}+B_{1}$ is a linear operator such that its restriction onto $A_{i}$ is a continuous operator into $B_{i}$ with operator norm $C_{i}$ for $i=0,1$, then its restriction onto $A_{\theta}=\left[A_{0}, A_{1}\right]_{\theta}$ is a continuous operator into $B_{\theta}=\left[B_{0}, B_{1}\right]_{\theta}$ with operator norm $C_{0}^{1-\theta} C_{1}^{\theta}$ for every $\theta \in(0,1)$.

(ii) For a measure space $\mathfrak{M}$ and $1<p_{0}, p_{1}<\infty$,

$$
\left[L_{p_{0}}\left(\mathfrak{M}, A_{0}\right), L_{p_{1}}\left(\mathfrak{M}, A_{1}\right)\right]_{\theta}=L_{p}\left(\mathfrak{M},\left[A_{0}, A_{1}\right]_{\theta}\right)
$$

for every $\theta \in(0,1)$, where $1 / p=(1-\theta) / p_{0}+\theta / p_{1}$.

(iii) For $m_{0}, m_{1} \in \mathbb{R}, 1<p_{0}, p_{1}<\infty$,

$$
\left[H_{p_{0}}^{m_{0}}, H_{p_{1}}^{m_{1}}\right]_{\theta}=H_{p}^{m},
$$

where $m=(1-\theta) m_{0}+\theta m_{1}$, and $1 / p=(1-\theta) / p_{0}+\theta / p_{1}$.

(iv) For $\theta \in[0,1]$ there is a constant $c_{\theta}$ such that

$$
|v|_{A_{\theta}} \leq c_{\theta}|v|_{A_{0}}^{1-\theta}|v|_{A_{1}}^{\theta}
$$

for all $v \in A_{0} \cap A_{1}$.

Now for an arbitrary $p \geq 2$ we take an integer $k \geq 1$ and a parameter $\theta \in[0,1]$ such that $p_{0}=2^{k} \leq p \leq 2^{k+1}=p_{1}$ and $1 / p=(1-\theta) / p_{0}+\theta / p_{1}$. By property (ii) we have

$$
\begin{gathered}
\Psi_{p}^{m}=\left[\Psi_{p_{0}}^{m}, \Psi_{p_{1}}^{m}\right]_{\theta}=H_{p}^{m}, \quad \mathbb{F}_{p}^{m}=\left[\mathbb{F}_{p_{0}}^{m}, \mathbb{F}_{p_{1}}^{m}\right]_{\theta}=L_{p}\left([0, T], H_{p}^{m}\right), \\
\mathbb{U}_{p}^{m}=\left[\mathbb{U}_{p_{0}}^{m}, \mathbb{U}_{p_{1}}^{m}\right]_{\theta}=L_{r}\left([0, T], H_{p}^{m}\right),
\end{gathered}
$$

and therefore by (i) the solution operator $\mathbb{S}$ is continuous for any $p \geq 2$ and integer $m \geq 0$, and the solution $u$ satisfies

$$
\left(\int_{0}^{T}|u(s)|_{H_{p}^{m}}^{r} d s\right)^{1 / r} \leq N\left(|\psi|_{H_{p}^{m}}+|f|_{\mathbb{U}_{p}^{m}}\right)
$$

with a constant $N=\left(p, d, m, T, K, K_{\bar{\eta}}, K_{\bar{\xi}}\right)$, where for any $s>0$ and $p>1, \mathbb{H}_{p}^{s}$ denotes the space of $H_{p}^{s}$-valued Borel functions $g$ on $[0, T]$ such that

$$
|g|_{\mathbb{H}_{p}^{s}}^{p}:=\int_{0}^{T}|g(t)|_{H_{p}^{s}}^{p} d t<\infty .
$$

Letting here $r \rightarrow \infty$ we obtain

$$
\operatorname{esssup}_{s \in[0, T]}|u(s)|_{H_{p}^{m}}^{p} \leq N^{p}\left(|\psi|_{H_{p}^{m}}^{p}+\int_{0}^{T}|f(s)|_{H_{p}^{m}}^{p} d s\right)
$$

with a constant $N=\left(p, d, m, T, K, K_{\bar{\eta}}, K_{\bar{\xi}}\right)$, which increases when $T$ increases.

The next theorem extends the above result to Eq. 1.1 with $\mathcal{R}$ satisfying Assumption 2.4. 
Theorem 5.4 Let Assumptions 2.1 through 2.5 hold with an integer $m \geq 1$ and a real number $p \geq 2$. Then Eq. 1.1 with initial condition $u(0)=\psi$ has a unique generalised solution $u \in \mathbb{W}_{p}^{m}$ such that estimate (5.17) holds.

Proof We use the standard method of continuity, see, e.g., [21]. For $\lambda \in[0,1]$, we consider the equation

$$
d u(t, x)=\left(\mathcal{A}^{0}(t) u(t, x)+\lambda \mathcal{R}(t) u(t, x)+f(t, x)\right) d t, \quad u(0, x)=\psi(x),
$$

for $x \in \mathbb{R}^{d}$, where

$$
\mathcal{A}^{0}(t)=\mathcal{L}(t)+\mathcal{M}(t)+\mathcal{N}(t)
$$

for every $t \in[0, T]$. Notice that Eq. 5.17 holds with any $t \in[0, T]$ in place of $T$. Hence, if $u \in \mathbb{W}_{p}^{m}$ is a generalised solution to Eq. 5.18, then by Assumption 2.4 we have

$$
\begin{gathered}
\underset{s \leq t}{\operatorname{esssup}}|u(s)|_{H_{p}^{m}}^{p} \leq N|\psi|_{H_{p}^{m}}^{p}+N \int_{0}^{t}|\lambda \mathcal{R}(s) u(s)|_{H_{p}^{m}}^{p}+|f(s)|_{H_{p}^{m}}^{p} d s \\
\leq C\left(|\psi|_{H_{p}^{m}}^{p}+|f|_{\mathbb{H}_{p}^{m}}^{p}+\int_{0}^{t} \underset{r \leq s}{\operatorname{esssup}}|u(r)|_{H_{p}^{m}}^{p} d s\right)
\end{gathered}
$$

with a constant $C=C\left(m, d, p, T, K, K_{\bar{\xi}}, K_{\bar{\eta}}\right)$. Hence by Gronwall's lemma we have estimate (5.17) for $u$. Let $\Lambda$ denote the set of $\lambda \in[0,1]$ such that for any $\psi \in W_{p}^{m}$ and $f \in \mathbb{W}_{p}^{m}$, (5.18) has a unique generalised solution in $\mathbb{W}_{p}^{m}$. Clearly $0 \in \Lambda$. Then we only need to prove $1 \in \Lambda$. To this end, it suffices to show that there is an $\delta>0$ such that for any $\lambda_{0} \in \Lambda$,

$$
\left[\lambda_{0}-\delta, \lambda_{0}+\delta\right] \cap[0,1] \in \Lambda \text {. }
$$

Fix $\lambda_{0} \in \Lambda, \psi \in W_{p}^{m}$ and $f \in \mathbb{W}_{p}^{m}$. For $v \in \mathbb{W}_{p}^{m}$ and $\lambda \in[0,1]$ we consider the equation $d u(t, x)=\left(\mathcal{A}^{0}(t) u(t, x)+\lambda_{0} \mathcal{R}(t) u(t, x)+\left(\lambda-\lambda_{0}\right) \mathcal{R}(t) v(t, x)+f(t, x)\right) d t, \quad u(0, x)=\psi(x)$ for $x \in \mathbb{R}^{d}$. Since $\lambda_{0} \in \Lambda$, this problem has a unique generalised solution $u \in \mathbb{W}_{p}^{m}$. Define the operator $Q_{\lambda}$ by $u=Q_{\lambda} v$. Then $Q_{\lambda}$ maps $\mathbb{W}_{p}^{m}$ into itself, and $\lambda \in \Lambda$ if and only if there is a fixed point of $Q_{\lambda}$. If $v_{i} \in \mathbb{W}_{p}^{m}$ and $u_{i}=Q_{\lambda} v_{i}$ for $i=1,2$, then for $u:=u_{2}-u_{1}$ we have

$$
d u(t)=\mathcal{A}^{0}(t) u(t)+\lambda_{0} \mathcal{R}(t) u(t)+\left(\lambda-\lambda_{0}\right) \mathcal{R}(t)\left(v_{2}(t)-v_{1}(t)\right), \quad u(0)=0 .
$$

Hence, using estimate (5.17) for $u$ and Assumption 2.4 on $\mathcal{R}$ we get

$$
\left|Q_{\lambda} v_{2}-Q_{\lambda} v_{1}\right|_{\mathbb{W}_{p}^{m}} \leq N^{\prime}\left|\lambda-\lambda_{0}\right|\left|\mathcal{R}\left(v_{2}-v_{1}\right)\right|_{\mathbb{W}_{p}^{m}} \leq N^{\prime \prime}\left|\lambda-\lambda_{0}\right|\left|v_{2}-v_{1}\right|_{\mathbb{W}_{p}^{m}},
$$

with constants $N^{\prime}$ and $N^{\prime \prime}$ depending only on $m, d, p, T, K, K_{\bar{\xi}}$ and $K_{\bar{\eta}}$. Taking $\delta=$ $\left(2 N^{\prime}\right)^{-1}$ we obtain that $Q_{\lambda}$ is a contraction mapping on $\mathbb{W}_{p}^{m}$ if $\lambda \in\left[\lambda_{0}-\delta, \lambda_{0}+\delta\right] \cap[0,1]$. Consequently, (5.18) has a unique solution $u$ in $\mathbb{W}_{p}^{m}$, and it satisfies (5.17).

When $s \in(0, m]$ is not an integer then we set $\theta=s-\lfloor s\rfloor$. Then by (ii) and (iii)

$$
\begin{gathered}
\Psi_{p}^{s}=\left[\Psi_{p}^{\lfloor s\rfloor}, \Psi_{p}^{\lceil s\rceil}\right]_{\theta}=H_{p}^{s}, \quad \mathbb{F}_{p}^{s}=\left[\mathbb{F}_{p}^{\lfloor s\rfloor}, \mathbb{F}_{p}^{\lceil s\rceil}\right]_{\theta}=L_{p}\left([0, T], H_{p}^{s}\right), \\
\mathbb{U}_{p}^{s}=\left[\mathbb{U}_{p}^{\lfloor s\rfloor}, \mathbb{U}_{p}^{\lceil s\rceil}\right]_{\theta}=L_{r}\left([0, T], H_{p}^{s}\right)
\end{gathered}
$$

for every $p \geq 2$ and integers $r>1$. We have seen above that under Assumptions 2.1, 2.2, 2.3 and 2.4 with $m \geq 1$, the solution operator $\mathbb{S}$ is continuous from $\Psi_{p}^{\lceil m\rceil} \times \mathbb{F}_{p}^{\lceil m\rceil}$ to $\mathbb{U}_{p, r}^{\lceil m\rceil}$, and from $\Psi_{p}^{\lfloor m\rfloor} \times \mathbb{F}_{p}^{\lfloor m\rfloor}$ to $\mathbb{U}_{p, r}^{\lfloor m\rfloor}$. Hence by (i) again for the solution $u$ we have

$$
\left(\int_{0}^{T}|u(t)|_{H_{p}^{s}}^{r} d t\right)^{1 / r} \leq N\left(|\psi|_{H_{p}^{s}}+|f|_{\mathbb{H}_{p}^{s}}\right)
$$


with a constant $N=\left(p, d, m, T, K, K_{\bar{\eta}}, K_{\bar{\xi}}\right)$. Letting here $r \rightarrow \infty$ we obtain

$$
\underset{t \in[0, T]}{\operatorname{esssup}}|u(t)|_{H_{p}^{s}} \leq N\left(|\psi|_{H_{p}^{s}}+|f|_{\mathbb{H}_{p}^{s}}\right) .
$$

By Lemma 3.12 we already know that the solution $u$ is in $C\left([0, T], H_{p}^{0}\right)$. To show that it is weakly continuous as an $H_{p}^{m}$-valued function we use the following lemma.

Lemma 5.5 Let $V$ be a reflexive Banach space, embedded continuously and densely into a Banach space $U$. Let $f$ be a $U$-valued weakly continuous function on $[0, T]$ and assume there is a dense subset $S$ of $[0, T]$ such that $f(s) \in V$ for $s \in S$ and $\sup _{s \in S}|f(s)|_{V}<\infty$. Then $f$ is a $V$-valued function, which is continuous in the weak topology of $V$.

Proof Since $S$ is dense in $[0, T]$, for a given $t \in[0, T]$ there is a sequence $\left\{t_{n}\right\}_{n=1}^{\infty}$ with elements in $S$ such that $t_{n} \rightarrow t$. Due to $\sup _{n \in \mathbb{N}}\left|f\left(t_{n}\right)\right|_{V}<\infty$ and the reflexivity of $V$ there is a subsequence $\left\{t_{n_{k}}\right\}$ such that $f\left(t_{n_{k}}\right)$ converges weakly in $V$ to some element $v \in V$. Since $f$ is weakly continuous in $U$, for every continuous linear functional $\varphi$ over $U$ we have $\lim _{k \rightarrow \infty} \varphi\left(f\left(t_{n_{k}}\right)\right)=\varphi(f(t))$. Since the restriction of $\varphi$ in $V$ is a continuous functional over $V$ we have $\lim _{k \rightarrow \infty} \varphi\left(f\left(t_{n_{k}}\right)\right)=\varphi(v)$. Hence $f(t)=v$, which proves that $f$ is a $V$-valued function. Moreover, by taking into account that

$$
|f(t)|_{V}=|v|_{V} \leq \liminf _{k \rightarrow \infty}\left|f\left(t_{n_{k}}\right)\right|_{V} \leq \sup _{t \in S}|f(t)|_{V}<\infty
$$

we obtain $K:=\sup _{t \in[0, T]}|f(s)|_{V}<\infty$. Let $\phi$ be a continuous linear functional over $V$. Due to the reflexivity of $V$, the dual $U^{*}$ of the space $U$ is densely embedded into $V^{*}$, the dual of $V$. Thus for $\phi \in V^{*}$ and $\varepsilon>0$ there is $\phi_{\varepsilon} \in U^{*}$ such that $\left|\phi-\phi_{\varepsilon}\right|_{V^{*}} \leq \varepsilon$. Hence

$$
\begin{aligned}
& \left|\phi(f(t))-\phi\left(f\left(t_{n}\right)\right)\right| \leq\left|\phi_{\varepsilon}\left(f(t)-f\left(t_{n}\right)\right)\right|+\left|\left(\phi-\phi_{\varepsilon}\right)\left(f(t)-f\left(t_{n}\right)\right)\right| \\
& \leq\left|\phi_{\varepsilon}\left(f(t)-f\left(t_{n}\right)\right)\right|+\varepsilon\left|f(t)-f\left(t_{n}\right)\right|_{V} \leq\left|\phi_{\varepsilon}\left(f(t)-f\left(t_{n}\right)\right)\right|+2 \varepsilon K .
\end{aligned}
$$

Letting here $n \rightarrow \infty$ and then $\varepsilon \rightarrow 0$, we get

$$
\limsup _{n \rightarrow \infty}\left|\phi(f(t))-\phi\left(f\left(t_{n}\right)\right)\right| \leq 0,
$$

which completes the proof of the lemma.

Clearly, $u$ is weakly continuous as an $H_{p}^{0}$-valued function. Hence applying Lemma 5.5 with $V=H_{p}^{m}$ and $U=H_{p}^{0}$, by using (5.20) with $s=m$, we obtain that $u$ is weakly continuous as an $H_{p}^{m}$-valued function. Thus by virtue of Eq. 5.20 we have

$$
\sup _{t \in[0, T]}|u(t)|_{H_{p}^{s}} \leq N\left(|\psi|_{H_{p}^{s}}+|f|_{\mathbb{H}_{p}^{s}}\right)
$$

for all $s \in[0, m]$ and $p \geq 2$ with a constant $N=N\left(m, p, d, K, K_{\bar{\xi}}, K_{\bar{\eta}}, T\right)$.

To show that $u$ is strongly continuous as an $H_{p}^{s}$-valued function for any $s<m$, notice that by the multiplicative inequality (iv) we have a constant $c$ such that for any sequence $t_{n} \rightarrow t$ in $[0, T]$ we have

$$
\left|u(t)-u\left(t_{n}\right)\right|_{H_{p}^{s}} \leq c\left|u(t)-u\left(t_{n}\right)\right|_{L_{p}}^{(m-s) / m}\left|u(t)-u\left(t_{n}\right)\right|_{H_{p}^{m}}^{s / m} .
$$

Letting here $n \rightarrow \infty$ we get $\lim _{n \rightarrow \infty}\left|u(t)-u\left(t_{n}\right)\right|_{H_{p}^{s}}=0$ by using (5.21) and the strong continuity of $u$ as an $L_{p}$-valued function. This shows that $u \in C\left([0, T], H_{p}^{s}\right)$ for every $s<m$ and finishes the proof of the existence of solutions in Theorem 2.1 for $V_{p}^{m}:=H_{p}^{m}$.

Consider now the case $V_{p}^{m}:=W_{p}^{m}$. Since for integers $m \geq 0$ the spaces $H_{p}^{m}$ and $W_{p}^{m}$ are the same as vector spaces equipped with equivalent norms for any $p \geq 1$, we need only 
consider the case when $m$ is not an integer and $p \geq 2$ is a real number. We will make use of the following facts about the interpolation spaces $\left(A_{0}, A_{1}\right)_{\theta, q}$ with parameters $\theta \in(0,1)$ and $q \in[1, \infty]$, obtained by real interpolation methods from an interpolation couple of Banach spaces $A_{0}$ and $A_{1}$ (see 1.3.3 in [43]).

(a) If $A_{0}, A_{1}$ and $B_{1}, B_{2}$ are two interpolation couples and $S: A_{0}+A_{1} \rightarrow B_{0}+B_{1}$ is a linear operator such that its restriction onto $A_{i}$ is a continuous operator into $B_{i}$ with operator norm $C_{i}$ for $i=0,1$, then its restriction onto $A_{\theta, q}=\left(A_{0}, A_{1}\right)_{\theta, q}$ is a continuous operator into $B_{\theta, q}=\left(B_{0}, B_{1}\right)_{\theta, q}$ with operator norm $C_{0}^{1-\theta} C_{1}^{\theta}$ for every $\theta \in(0,1)$ and $q \in[1, \infty]$.

(b) For a $\sigma$-finite measure space $\mathfrak{M}$ for $p_{0}, p_{1} \in(1, \infty)$ we have

$$
\left(L_{p_{0}}\left(\mathfrak{M}, A_{0}\right), L_{p_{1}}\left(\mathfrak{M}, A_{1}\right)\right)_{\theta, p}=L_{p}\left(\mathfrak{M},\left(A_{0}, A_{1}\right)_{\theta, p}\right)
$$

for every $\theta \in(0,1)$, where $1 / p=(1-\theta) / p_{0}+\theta / p_{1}$.

(c) For $s_{0}, s_{1} \in(0, \infty), s_{0} \neq s_{1}$

$$
\left(W_{p}^{s_{0}}, W_{p}^{s_{1}}\right)_{\theta, p}=W_{p}^{s} \quad \text { for } \theta \in(0,1) \text { and } p \in(1, \infty)
$$

when $s:=(1-\theta) s_{0}+\theta s_{1}$ is not an integer.

(d) For $\theta \in(0,1)$ and $q \in[1, \infty]$ there is a constant $c_{\theta, q}$ such that

$$
|v|_{A_{\theta, q}} \leq c_{\theta, q}|v|_{A_{0}}^{1-\theta}|v|_{A_{1}}^{\theta}
$$

for all $v \in A_{0} \cap A_{1}$.

For a fixed $t \in[0, T]$ consider the operator $\mathbb{S}(t)$ mapping $(\psi, f) \in W_{p}^{n} \times L\left([0, T], W_{p}^{n}\right)$ to $u(t) \in W_{p}^{n}$, the solution of Eq. 1.1 at time $t$. We already know that $\mathbb{S}(t)$ is a bounded operator for $p \geq 2$ and integers $n \in[0, m]$, and its norm can be estimated by the right-hand side of Eq. 5.21 in this case. When $n=s \geq 0$ is not an integer, then we set $\theta=s-\lfloor s\rfloor$, and using (b) and (c) we have

$$
\left[W_{p}^{\lfloor s\rfloor}, W_{p}^{\lceil s\rceil}\right]_{\theta, p}=W_{p}^{s}, \quad\left[L_{p}\left([0, T], W_{p}^{\lfloor s\rfloor}\right), L_{p}\left([0, T], W_{p}^{\lceil s\rceil}\right)\right]_{\theta, p}=L_{p}\left([0, T], W_{p}^{s}\right),
$$

Moreover by (a) we get that $u(t) \in W_{p}^{s}$ for every $t \in[0, T]$ and $s \in[0, m]$, and we have

$$
\sup _{t \in[0, T]}|u(t)|_{W_{p}^{s}}^{p} \leq N|\psi|_{W_{p}^{s}}^{p}+N \int_{0}^{T}|f(t)|_{W_{p}^{s}}^{p} d t
$$

for every $s \in[0, m]$ and $p \geq 2$. Hence taking into account that $u$ is strongly continuous in $t$ as an $L_{p}$-valued function, by (c) and (d) we get that it is (strongly) continuous as a $W_{p^{-}}^{s^{-}}$ valued function for every $s<m$. Moreover, using Lemma 5.5 with $V=W_{p}^{m}$ and $U=L_{p}$ it follows that $u$ is weakly continuous as a $W_{p}^{m}$-valued function.

Acknowledgments A generalisation of Theorem 2.1 to stochastic integro-differential equations was presented at the conference on "Harmonic Analysis for Stochastic PDEs" in Delft, 10-13 July, 2018 and at the "9th International Conference on Stochastic Analysis and Its Applications" in Bielefeld, 3-7 September, 2018. The authors are grateful to the organisers of these conferences for the invitation and for discussions. They thank Stefan Geiss and Mark Veraar for useful discussions and Alexander Davie for correcting some mistakes. The authors are also thankful to the referees for their comments which helped to improve the presentation of the paper.

Open Access This article is licensed under a Creative Commons Attribution 4.0 International License, which permits use, sharing, adaptation, distribution and reproduction in any medium or format, as long as you give appropriate credit to the original author(s) and the source, provide a link to the Creative Commons licence, and indicate if changes were made. The images or other third party material in this article are included in the article's Creative Commons licence, unless indicated otherwise in a credit line to the material. If 
material is not included in the article's Creative Commons licence and your intended use is not permitted by statutory regulation or exceeds the permitted use, you will need to obtain permission directly from the copyright holder. To view a copy of this licence, visit http://creativecommonshorg/licenses/by/4.0/.

\section{References}

1. Applebaum, D.: Lévy processes and stochastic calculus. Cambridge University Press, Cambridge (2009)

2. Barndorff-Nielsen, O.E., Mikosch, T., Resnick, S.I. (eds.): Lévy Processes. Theory and Applications. Birkhäuser, Cambridge (2001)

3. Bucur, C., Valdinoci, E.: Nonlocal diffusion and applications, lecture notes of the unione matematica italiana. Springer International Publishing, Switzerland (2016)

4. Caffarelli, L., Silvestre, L.: An extension problem related to the fractional Laplacian. Commun. Partial Differ. Equ. 32(7-9), 1245-1260 (2007)

5. Caffarelli, L., Salsa, S., Silvestre, L.: Regularity estimates for the solution and the free boundary of the obstacle problem for the fractional Laplacian. Inventiones Math. 171(2), 425-461 (2008)

6. Chang, T., Lee, K.: On a stochastic partial differential equation with a fractional Laplacian operator. Stoch. Process. Appl. 122(9), 3288-3311 (2012)

7. Chen, Z., Kim, K.: An $L_{p}$-theory for non-divergence form SPDEs driven by Lévy processes. Forum Mathematicum 26(5), 1381-1411 (2014)

8. Cont, R., Tankov, P.: Financial modelling with jump processes. Chapman \& Hall CRC Press Company, London (2004)

9. Dareiotis, K.: A note on degenerate stochastic integro-differential equations arXiv:1406.5649v1 (2014)

10. Freidlin, M.I.: On the factorization of nonnegative definite matrices. Teor. Veroyatn. Primen. 33(2), 375-378 (1968)

11. Gordon, W.B.: On the diffeomorphisms of euclidean space. Am. Math. Monthly 79(7), 755-759 (1972)

12. Garroni, M.G., Menaldi, J.L.: Second order elliptic integro-differential problems. Chapman \& Hall CRC Press Company, London (2002)

13. Garroni, M.G., Menaldi, J.L.: Green functions for second order parabolic integro-differential problems. Longman, UK (1992)

14. Gerencsér, M., Gyöngy, I.: Finite difference schemes for stochastic partial differential equations in Sobolev spaces. Appl. Math. Optim. 72(1), 77-100 (2015)

15. Gerencsér, M., Gyöngy, I., Krylov, N.V.: On the solvability of degenerate stochastic partial differential equations in Sobolev spaces. Stoch. Partial Differ. Equ. Anal. Comput. 3(1), 52-83 (2015)

16. Ikeda, N., Watanabe, S.: Stochastic differential equations and diffusion processes North-Holland (2011)

17. Jacob, N.: Pseudo-Differential Operators and markov processes: generators and potential theory imperial college press (2002)

18. Krylov, N.V.: On $L_{p}$-theory of stochastic partial differential equations in the whole space. SIAM J. Math. Anal. 27(2), 313-340 (1996)

19. Kim, K., Kim, P.: An $L_{p}$-theory of a class of stochastic equations with the random fractional Laplacian driven by lévy processes. Stoch. Process. Appl. 122(12), 3921-3952 (2012)

20. Kim, K., Lee, K.: A note on $W_{p}^{\gamma}$-theory of linear stochastic parabolic partial differential systems. Stoch. Process. Appl. 123, 76-90 (2013)

21. Krylov, N.V.: Lectures on elliptic and parabolic equations in sobolev spaces graduate studies in mathematics, vol. 96. AMS, Providence, Rhode Island (2008)

22. Krylov, N.V.: Itô's formula for the $L_{p}$-norm of stochastic $W_{p}^{1}$-valued processes. Probab. Theory Relat. Fields 147, 583-605 (2010)

23. Krylov, N.V., Rozovskii, B.L.: On the Cauchy problem for linear stochastic partial differential equations. Math. USSR Izvestija 11(6), 1267-1284 (1977)

24. Krylov, N.V., Rozovskii, B.L.: Characteristics of degenerating second-order parabolic Itô equations. J. Soviet Maths. 32, 336-348 (1986). (Translated from Trudy Seminara imeni I.G. Petrovskogo, No. 8. pp.. 153-168, 1982.)

25. Leahy, J.-M., Mikulevicius, R.: On classical solutions of linear stochastic integro-differential equations. Stoch. Partial Differ. Equ. Anal. Comput. 4(3), 535-591 (2016)

26. Leahy, J.-M., Mikulevicius, R.: On degenerate linear stochastic evolution equations driven by jump processes. Stoch. Process. Appl. 125(10), 3748-3784 (2015)

27. Mikulevičius, R., Phonsom, C.: On $L_{p}$-theory for parabolic and elliptic integro-differential equations with scalable operators in the whole space. Stoch. Partial Differ. Equ. Anal. Comput. 5(4), 472-519 (2017) 
28. Mikulevičius, R., Phonsom, C.: On the Chauchy problem for stochastic integro-differential parabolic equations in the scale of $L_{p}$-spaces of generalised smoothness arXiv:1805.03232v1 (2018)

29. Mikulevičius, R., Pragarauskas, H.: On the Cauchy problem for certain integro-differential operators in Sobolev and Hölder spaces. Liet. Mat. Rink. 32(2), 299-331 (1992). translation in Lithuanian Math. J. 32 (1992), no.2 238-264 (1993)

30. Mikulevičius, R., Pragarauskas, H.: On Classical Solutions of Certain Nonlinear Integro-Differential Equations. Stochastic Processes and Optimal Control (Friedrichroda, 1992), 151-163 Stochastics Monogr., vol. 7. Gordon and Breach, Montreux (1993)

31. Mikulevicius, R., Pragarauskas, H.: On $L_{p}$-theory for stochastic parabolic integro-differential equations. Stoch. Partial Differ. Equ. Anal. Comput. 1(2), 282-324 (2013)

32. Mikulevičius, R., Xu, F.: On the Cauchy proble for parabolic integro-differential equations in generalised Hölder spaces, arXiv:1806.07019v1 (2018)

33. Murray, J.D. Mathematical biology: I. An Introduction, 3rd edn. Springer, New York (2007)

34. Oleı̆nik, O.A.: Alcuni risultati sulle equazioni lineari e quasi lineari ellittico-paraboliche a derivate parziali del secondo ordine, (Italian). Atti Accad. Naz. Lincei Rend. Cl. Sci. Fis. Mat Natur. 40(8), 775-784 (1966)

35. Oleĭnik, O.A.: On the smoothness of solutions of degenerating elliptic and parabolic equations. Dokl Akad. Nauk SSSR 163, 577-580 (1965). in Russian; English translation in Soviet Mat. Dokl. Vol, 6 (1965), No. 3, 972-976

36. Oleı̆nik, O.A., Radkevič, E.V.: Second order equations with nonnegative characteristic form, Mathematical Analysis, 1969, pp. 7-252. (errata insert) Akad. Nauk SSSR, Vsesojuzn. Inst. Naučn. i Tehn. Informacii, Moscow, 1971 in Russian, English translation: Plenum Press New York-London (1973)

37. Olejnik, O.A., Radkevich, E.V.: Second order equations with nonnegative characteristic form, AMS, Providence (1973)

38. Pascucci, A.: PDE And martingale methods in option pricing. Springer, Italia (2011)

39. Pragarauskas, G.: The first boundary value problem for a certain class of integro-differential equations. Litovsk. Mat. Sb. 14(4), 195-200 (1974)

40. Pragarauskas, G.: On the Bellman equation for weakly degenerate general random processes. Litovsk. Mat. Sb. 20(2), 129-136 (1980)

41. Rozovskii, B.L., Systems, S.tochastic.E.volution.: Linear Theory and Applications to Nonlinear Filtering. Kluwer, Dordrecht (1990)

42. Stinga, P.R., Torrea, J.L.: Extension problem and Harnack's inequality for some fractional operators. Comm. Partial Differ. Equ. 35, 2092-2122 (2010)

43. Triebel, H.: Interpolation Theory - Function Spaces - Differential Operators, North holland publishing company Amsterdam-New York-Oxford (1978)

Publisher's Note Springer Nature remains neutral with regard to jurisdictional claims in published maps and institutional affiliations. 